九州大学学術情報リポジトリ

Kyushu University Institutional Repository

\title{
Molecular Analyses of an Unusual Translesion DNA Polymerase from Methanosarcina acetivorans C2A
}

Lin, Li-Jung

Department of Animal Sciences, University of Illinois at Urbana-Champaign

Yoshinaga, Aya

Department of Genetic Resource Technology, Kyushu University

Lin, Yuyen

Department of Animal Sciences, University of Illinois at Urbana-Champaign

Guzman, Claudia

Department of Animal Sciences, University of Illinois at Urbana-Champaign

他

http://hdl. handle. net/2324/26622

出版情報：Journal of Molecular Biology. 397 (1)，pp.13-30，2010-03-19. Elsevier バージョン:

権利関係: (C) 2010 Elsevier Ltd. 


\section{Molecular analyses of an unusual translesion DNA polymerase from Methanosarcina acetivorans C2A}

Li-Jung Lin ${ }^{1}$, Aya Yoshinaga ${ }^{4}$, Yuyen Lin ${ }^{1}$, Claudia Guzman ${ }^{1}$, Yi-Hsing Chen ${ }^{1}$, Shou Mei ${ }^{7}$, Angelica M. Lagunas ${ }^{1}$, Satoshi Koike ${ }^{5}$, Shigenori Iwai ${ }^{6}$, M. Ashley Spies ${ }^{2,3}$, Satish K. Nair ${ }^{2,3}$, Roderick I. Mackie ${ }^{1,3}$, Yoshizumi Ishino ${ }^{4 *}$, and Isaac K. O. Cann ${ }^{1,3,7, *}$

From the Department of Animal Sciences ${ }^{1}$, Department of Biochemistry ${ }^{2}$, Institute for Genomic Biology ${ }^{3}$ Department of Microbiology ${ }^{7}$, University of Illinois at UrbanaChampaign, Urbana, IL 61801, USA. Kyushu University ${ }^{4}$, Hokkaido University ${ }^{5}$, Osaka University ${ }^{6}$, Japan

Running title: DNA polymerase, translesion, DNA synthesis, archaea, PCNA

*To whom correspondence should be addressed.

\section{Dr. Yoshizumi Ishino}

or

\section{Dr. Isaac K.O. Cann}

Department of Animal Sciences, 1207 West Gregory Drive, University of Illinois at Urbana-Champaign, IL 61801,

Tel. 217-333-2090,

Fax, 217-333-8286

E-mail: icann@uiuc.edu 


\section{SUMMARY}

The domain Archaea is composed of several subdomains, and prominent among them are the Crenarchaeota and the Euryarchaeota. Biochemically characterized archaeal family Y DNA polymerases or DinB homologs, to date, are all from crenarchaeal organisms, especially the genus Sulfolobus. Here, it is demonstrated that archaeal family $Y$ DNA polymerases fall into five clusters based on phylogenetic analysis. The homolog from the euryarchaeon Methanosarcina acetivorans, designated MacDinB-1 and characterized in this study, belongs to cluster II. Therefore, MacDinB-1 is different from the Sulfolobus DinB proteins, which are members of cluster I. In addition to translesion DNA synthesis activity, MacDinB-1 synthesized unusually long products $(\sim 7.2 \mathrm{~kb})$ in the presence of its cognate Proliferating Cell Nuclear Antigen (PCNA). The PCNA-interacting site in MacDinB-1 was identified by mutational analysis in a C-terminally located heptapeptide akin to a PCNA interacting protein (PIP) box. In vitro assays from the present report suggested that MacDinB-1 works in an error-free mode to repair cyclobutane pyrimidine dimers (CPD). This study on a euryarchaeal DinB homolog provides important insights into functional diversity of the family Y DNA polymerases, and the availability of a genetic system for this archaeon should allow subsequent elucidation of the physiological significance of this enzyme in $M$. acetivorans cells.

KEY WORDS: translesion DNA synthesis, DinB, archaea, family Y polymerase, PCNA 


\section{INTRODUCTION}

All organisms exposed to the sun experience UV radiation, a potent DNA damaging agent known to cause DNA lesions such as cis-syn thymine-thymine (TT) dimers and 6-4 photoproducts ${ }^{1}$. Although replicative DNA polymerases are equipped with highly accurate active sites and proofreading activities that ensure high fidelity DNA synthesis, most of these polymerases are incapable of replicating through UV-induced lesions ${ }^{2 ; 3}$. Thus, these lesions, if unrepaired, impede progress of a growing DNA replication fork. How the DNA polymerases acting at the replication fork overcome these blocking lesions was perplexing until the discovery of specialized DNA polymerases collectively called the $\mathrm{Y}$ family DNA polymerases 4; 5; 6 . Subsequently, these polymerases were shown to be present in cells across the three domains of life ${ }^{4 ; 7}$. The family Y DNA polymerases carry out translesion DNA synthesis (TLS), and thus they are thought to be recruited to temporarily replace the high-fidelity replicative polymerases in order to replicate through DNA lesions, such as those generated by UV radiation $^{8 ; 9 ; 10}$.

The bacterium Escherichia coli possesses two family Y DNA polymerases, namely PollV (DinB) and PolV (UmuD' $\left.{ }_{2} \mathrm{C}\right)$. Although they belong to the same family, $E$. coli PolIV and PolV appear to perform distinct physiological functions. PolV is clearly the major lesion bypass polymerase involved in damage-induced mutagenesis $5 ; 11$. The recombinase RecA, the ssDNA-binding protein $\mathrm{SSB}$, and the processivity factor $\beta$ clamp act as accessory proteins for PolV-mediated error-prone translesion DNA synthesis $1 ; 4 ; 5$;

12. Thus, in the presence of activated RecA protein, SSB, $\beta$-clamp and its loader $(\gamma$ complex), PolV can bypass multiple template lesions, including TT (6-4) photoproduct, 
TT cis-syn cyclobutane pyrimidine dimer (CPD), and abasic sites ${ }^{1 ; 3}$. In contrast, although E. coli PolIV, under the same conditions, incorporated nucleotides opposite the lesions, it exhibited no further extension ${ }^{1}$. Furthermore, it has been shown that both PolV and PolIV are required for either error-free or -1 frame shift translesion synthesis at a benzo(a)pyrene adduct ${ }^{13}$.

In eukaryotes, several Y family polymerases have been identified in human cells

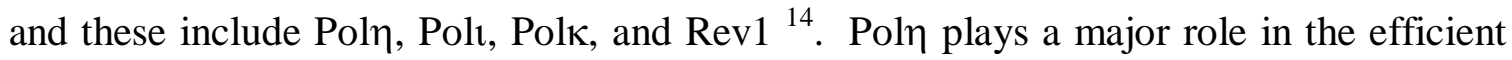
bypass of CPD sites and inserts the correct bases opposite the lesion ${ }^{15}$. The function of Polt in vivo is not clear ${ }^{14}$. In in vitro experiments, Polı was able to insert bases opposite several types of lesions in a highly error prone fashion, and it exhibited a very limited capacity to extend DNA beyond the inserted bases ${ }^{16}$. Polk, which is the homolog of the DinB proteins, has been suggested to be important in nucleotide excision repair (NER), although based on in vitro analysis there is also evidence that in NER, DNA synthesis may be carried out by the replicative DNA polymerases $\delta$ and $\varepsilon{ }^{14}$. Rev1, which exhibits limited homology to the E. coli UmuC protein, is a deoxycytidyl transferase and can transfer dCMP from dCTP to the 3' end of a primer in a template dependent fashion ${ }^{17}$.

TLS in Archaea, which constitutes a third domain of life, has served as an important paradigm by providing significant insights into the molecular mechanisms of lesion bypass ${ }^{18 ; 19 ; 20}$. Archaea are divided into several subdomains. However, cultivable organisms are mostly from the crenarchaeal and euryarchaeal subdomains, and the investigations on TLS have focused on the thermostable Y-family polymerases of the crenarchaeal genus Sulfolobus. Thus, knowledge on TLS DNA polymerases of mesophilic archaea and also of the subdomain Euryarchaeota is lacking. We identified 
two genes encoding DinB-like DNA polymerases in the genomes of two euryarchaea: Methanosarcina. mazei and M. barkeri. However, only one of such genes could be found in the other Methanosarcina species, M. acetivorans C2A. A critical examination of the genome of $M$. acetivorans showed a clear degeneration of the gene encoding the second DinB homolog (Lin et al. unpublished). Phylogenetic analysis showed that the two DinB homologs (DinB-1 and DinB-2) in the Methanosarcinales fall into two different clusters, which may also suggest different biochemical properties between the two enzymes. The preservation of DinB-1 and the loss of DinB-2 in the genome of $M$. acetivorans $\mathrm{C} 2 \mathrm{~A}$ may also suggest DinB-1 as more critical to the integrity of the genomes of the Methanosarcinales. In an effort to understand the biochemistry of DinB-1, we have cloned the gene for DinB-1 from the $M$. acetivorans C2A genome, and characterized the gene product, MacDinB-1, produced in E. coli cells. We found that MacDinB-1 interacts with its cognate proliferating cell nuclear antigen (PCNA) at the C-terminally located PIP ( PCNA interacting protein) - box. To our surprise, MacDinB synthesized distinctly long $(\sim 7.2 \mathrm{~kb})$ DNA products in the presence of PCNA in vitro. This finding is in contrast to the results reported for other archaeal DinB homologs ${ }^{9}$, and also different from a DinB homolog from group $\mathrm{V}$ analyzed in the present study. It is anticipated that results obtained from this work will provide important insights into the function and properties of the proteins that cluster with MacDinB-1, and subsequent detailed research on the DinB-2 homologs will clarify the need for two DinB homologs and how they share functions in some Methanosarcinales. 


\section{RESULTS}

\section{Phylogenetic analysis of archaeal family Y DNA polymerases}

Because of the rapid accumulation of genomic sequences in the databases, we were able to retrieve 30 genes encoding DinB homologs in archaeal genomes to investigate their evolutional relationships. The unrooted phylogenetic tree in Fig. 1 suggested that the archaeal DinB proteins group into five clusters. Cluster I comprises the DinB homologs of the hyperthermophilic crenarchaeotes. Among these are the Sulfolobus spp Dpo4 and the Dbh, which are the most well characterized DinB homologs in the archaeal domain ${ }^{9 ;}$ 18; 19; 31 . In cluster II is the group designated DinB-1 of the Methanosarcinales, members of which include the Methanosarcina spp and Methanococcoides burtonii. There are only two representatives in cluster III, and each protein comes from members of the socalled mesophilic crenarchaeotes, a group with only one cultured member, Nitrosopumilus maritimus $^{32}$. Cluster IV comprises DinB homologs from the haloarchaea, which are known to exist in very high salt environments, and in cluster V are the DinB-2 homologs of the Methanosarcinales and also DinB proteins from other euryarchaeotes, mostly methanogens. From the topology of the tree, the DinB proteins can also be clearly divided into the crenarchaeal cluster (cluster I) and the euryarchaeal cluster (Clusters II, III, IV, and V). The grouping of the proteins from the mesophilic crenarchaeotes ( $N$. maritimus and $C$. symbosium) with the proteins from the euryarchaea is not surprising, since their genomes also contain genes such as $d p 1$ and $d p 2$, encoding DNA polymerase D (PolD), which had hitherto been found only in the euryarchaea ${ }^{33 ; 34 ;}$ 35. In fact, these organisms have been suggested as being different from the crenarchaeotes, and that they belong to a new subdomain with Thaumarchaeota as their 
proposed name ${ }^{36}$. Grouping with the crenarchaeal cluster are also Picrophilus torridus and Ferroplasma acidarmanus DinB homologs. Although the two organisms are members of the Euryarchaeota, it has been suggested that by sharing similar habitats, there has been significant exchange of genetic information between their lineage (Thermoplasmatales) and the Sulfolobales ${ }^{37}$.

Based on the alignment of the archaeal DinB polypeptide sequences, we could identify the five motifs described for the DinB family of proteins ${ }^{6}$. In addition, we propose a new motif, motif VI, found at the extreme C-termini of these polypeptides (Fig. 2). The sequence of motif VI is similar to a conserved motif called PCNA-interacting protein (PIP) box, QXXhXXaa, where $X$ represents any amino acid, $h$ represents hydrophobic residues (e.g. Leu, Ile, or Met), and $a$ represents aromatic residues (e.g. Phe, Tyr, or Trp), and therefore, the motif was predicted to mediate interaction with the archaeal homolog of the eukaryotic proliferating cell nuclear antigen (PCNA). The evidence verifying this hypothesis is provided in this report as described below.

\section{Purification of proteins}

The DinB-1 polymerase of $M$. acetivorans was purified by affinity chromatography and anion exchange chromatography as described in the Materials and Methods. The cognate processivity factor PCNA or sliding clamp was purified by Polymin-P treatment, ammonium sulfate precipitation and anion exchange chromatography, and the clamp loader or RFC was purified by affinity, heparin and size exclusion chromatographies. As shown in Figure 3, we successfully purified MacDinB-1 and MacPCNA from the recombinant E. coli cells (lanes 2, and 4). To load PCNA onto DNA, when a circular 
ssDNA template was used, we produced the cognate replication factor C (RFC) (Fig. 3, lane 5). In lane 3 is a MacDinB-1 derivative with motif VI deleted for use in investigating MacDinB-1/MacPCNA interaction. As can be seen from Fig. 3, each protein was purified to near homogeneity.

\section{Translesion DNA synthesis by MacDinB-1}

MacDinB-1 was tested for its capacity to synthesize DNA past DNA lesions using a primer annealed to a linear DNA (49mer) template. A simple illustration of the substrates is provided in Fig. 4A. As shown in Fig 4B, by increasing the concentration of MacDinB-1 in the reaction mixture with non-damaged DNA as the template, the labeled 27-mer oligonucleotide was increasingly extended, until a product of approximate size 49-mer became dominant at $200 \mathrm{nM}-500 \mathrm{nM}$ protein concentrations. We, therefore, used a protein concentration of $500 \mathrm{nM}$ to determine whether MacDinB-1 can synthesize through three different DNA lesions. The lesions were abasic (Ab) DNA, CPD, and (6-4) photoproduct. By incubating MacDinB-1 with each of the three lesions, we observed that a larger product, compared to the 27-mer primer, was synthesized for both abasic DNA and CPD templates (Fig. 4C). Under the same experimental conditions, we did not observe DNA synthesis through the (6-4) photoproduct lesion.

A possible insertion sequence (DPGENG, Fig. 2 motif III) is seen in the Methanosarcinal DinB-1. Interestingly, deletion of the insertion sequence from MacDinB-1 did not yield any obvious difference from the results obtained for the wild type protein (results not shown). 


\section{MacDinB-1 preferentially incorporates " $A$ " at lesion sites}

After determining that MacDinB-1 can carry out translesion DNA synthesis, its nucleotide preference for incorporation at damaged DNA sites was investigated. Initially we tested incorporation on non-damaged DNA. By using a template that required an A as the incoming nucleotide, addition of all 4 dNTP's (G, A, T, C) resulted in synthesis of longer products of different sizes (Fig. 5A, lane 2). When we restricted the nucleotides in the reaction mixture to individual nucleotides, the majority of the incorporated products were two A's. This was the expected product (Fig. 5A, lane 4). Under these conditions, we did not observe misincorporation by MacDinB-1 (Fig. 5A, lanes 3, 5, and 6). In Fig. $5 \mathrm{~B}$, it was observed that upon encountering an abasic site, MacDinB-1 preferred to incorporate the nucleotide A. The results shown in the lane with all 4 dNTP's (Fig. 5B lane 2) also show that there is a pause by the polymerase upon incorporating the first base. However, longer products were still made under these conditions. In Fig. 5C, it was observed that the correct nucleotides (2A's) were incorporated opposite a CPD lesion. Here also, the polymerase exhibited a pause in the presence of all 4 dNTPs (Fig. 5C lane 2). Compared with the products in Fig. 5C lane 4, the pause most likely occurs after incorporation of the first nucleotide. However, the polymerase was still able to synthesize longer products.

\section{MacPCNA stimulates translesion DNA synthesis by MacDinB-1}

At the extreme C-terminal region of MacDinB-1 (Fig. 2, motif VI) is a short sequence

(QTSLFDY) with similarity to the so-called PCNA interacting protein (PIP)-box ${ }^{38}$. Based on this information, we determined whether translesion DNA synthesis by 
MacDinB-1 is stimulated by its cognate sliding clamp or MacPCNA. As shown in Fig 6, as we kept MacDinB-1 concentration constant and increased MacPCNA concentration, DNA synthesis on the abasic DNA (Fig. 6A) and CPD (Fig. 6B) templates were each stimulated and longer products of approximately 49-mer were synthesized. Whereas the pause seen on CPD (Fig. 5C lane 2) was absent in the presence of PCNA (Fig. 6B), the one seen with the abasic DNA lesion persisted (Fig. 6A). We did not see stimulation of DNA synthesis through the (6-4) photoproduct lesion in the presence of MacPCNA (results not shown).

\section{MacDinB-1 interacts with MacPCNA}

The stimulation of MacDinB-1 polymerase activity by MacPCNA and the presence of a PIP-box-like sequence (QTSLFDY) at the C-terminal region of MacDinB-1 led us to investigate whether a physical interaction between the two proteins can be detected by gel filtration analysis. Highly purified MacDinB-1 and MacPCNA samples were either injected individually or as a mixture to a gel filtration column. The estimated molecular mass of MacDinB-1, based on its polypeptide sequence, is $42.3 \mathrm{kDa}$ and the elution volume $(15.0 \pm 0.1 \mathrm{ml})$ from the gel filtration analysis (Fig. 7Ai) suggested a protein of molecular mass $42.7 \pm 0.6 \mathrm{kDa}$. The results thus suggested that MacDinB-1 is a monomer in solution. The elution volume $(13.9 \pm 0.1 \mathrm{ml})$ of MacPCNA (Fig. 7Aii) suggested a

protein of molecular mass $80.6 \pm 5.2 \mathrm{kDa}$. Since the estimated molecular mass of a monomer of MacPCNA, based on its polypeptide sequence, is $26.6 \mathrm{kDa}$, the results suggested a protein existing as a homotrimer in solution. The shoulder observed in the elution profile (Fig. 7Aii) may represent other oligomerization states of MacPCNA, for 
example a dimer as described elsewhere ${ }^{33}$. When the dialysate of MacDinB1/MacPCNA mixture was analyzed by gel filtration, the two proteins co-eluted at an elution volume of $(12.6 \pm 0.1 \mathrm{ml})$, which suggested a complex of molecular mass 163.1 kDa (Fig. 7Aiii). Thus, the two proteins formed a stable complex in solution. Based on the molecular masses of the individual proteins, as stated above, we estimated a ratio of 1:2, i.e., 1 mole of MacPCNA homotrimer forms a complex with 2 moles of MacDinB-1, although we anticipated three possible binding sites in the homotrimeric MacPCNA. On the other hand, a dialysate of a MacDinB-1 derivative with the putative PIP-box deleted (MacDinB-1 $\Delta \mathrm{T}$ ) eluted as two peaks, with the first peak representing MacPCNA and the second peak representing MacDinB-1 $\Delta \mathrm{T}$ (Fig. 7Aiv). Therefore, deletion of the PIP-boxlike sequence at the C-terminus of MacDinB-1 abolishes interaction with PCNA, indicating that this peptide is a functional PIP-box. The lack of physical interaction between MacDinB-1 $\Delta \mathrm{T}$ and MacPCNA is also discernible from the corresponding SDSPAGE of aliquots of fractions from the gel filtration analysis (Fig. 7B). Interaction of MacDinB-1 and MacPCNA shifted the two bands to earlier elution volumes (MacDinB1/MacPCNA panel), whereas a shift of individual protein bands was not seen in the case of the MacDinB-1 1 T/MacPCNA panel.

\section{Unique mutations in PIP-box impair stimulation of MacDinB-1 DNA synthesis}

We examined the putative PIP-boxes of several polypeptide sequences of $M$. acetivorans proteins shown in our lab to interact with MacPCNA (MacDinB-1, MacPolBI-I, MacPolBI-II, and MacFEN-I) or predicted to interact with MacPCNA (MacRFCL-I, MacRFCL-II, and MacDP2) ${ }^{33 ; 35}$. An alignment of the putative PIP-boxes and those 
from two human proteins known to interact with human PCNA is shown in Fig. 8. Note that some archaeal PIP-box like sequences are composed of seven instead of the eight residues originally proposed by Warbrick ${ }^{38}$. Clearly, some residues at certain positions in this motif are highly conserved. This is especially true for the glutamine at position 1 , the leucine at position 4, the aspartate at position 6 , and the phenylalanine at position 7 . Each of the amino acid residues in the PIP-box of MacDinB-1 was, therefore, mutated to alanine to determine the effect on DNA synthesis. In the DNA synthesis experiment, we used M13mp18 circular ssDNA $(\sim 7.2 \mathrm{~kb})$ as the template and this required the presence of MacRFC as the cognate clamp loader in the reaction mixture to load MacPCNA onto the DNA template. Surprisingly, as shown in Fig 9A (lane 1, WT), MacDinB-1 completely replicated the entire phage genome in the presence of the cognate PCNA and RFC. Deleting the PIP-box from MacDinB-1 abolished this capacity to synthesize long products in the presence of accessory factors (Fig. 9A, lane $\Delta$ ). We also found that mutations that converted three different residues to alanine (Gln to Ala or Leu to Ala or Tyr to Ala) in the PIP-box sequence impaired the capacity to carry out long DNA synthesis even in the presence of MacPCNA (Fig. 9A, lanes representing Q, L, and Y). In contrast, mutating each of the four remaining residues (Thr to Ala or Ser to Ala or Phe to Ala or Asp to Ala) in the PIP-box had little (Ser to Ala) to no effect on the capacity to synthesize long DNA in the same reaction condition (lanes representing T, S, F and D). The analyses of the impaired mutants were also presented in comparison with the wild type MacDinB-1 with and without the accessory proteins (Supplementary Fig.1). To visualize the products in Fig. 9A in greater detail, we resolved each of the products on acrylamide gel (Fig. 9B). Here it was clear that deleting the PIP-box $(\Delta)$ or changing 
each of the Gln, Leu, and Tyr in the PIP-box to Ala resulted in a MacDinB-1 derivative that was more distributive in DNA synthesis, which was similar to synthesis by MacDinB-1 alone or in the absence of either MacPCNA or MacRFC (Fig. 9C), although in this case the products were shorter.

\section{Interactions of MacDinB-1 PIP-box mutants with MacPCNA}

To reconcile our findings above with interactions with the sliding clamp, we determined how each mutation in the MacDinB-1 PIP-box impacted its interaction with MacPCNA. As shown in Fig. 10A, in the first panel, MacDinB-1 co-eluted with MacPCNA during gel filtration analysis (DinB WT/PCNA). Deletion of the PIP-box ( $\triangle$ PIP-box) led to failure of this derivative ( $\triangle$ PIP-box) to co-elute with the cognate PCNA (panel 2, DinB $\Delta /$ PCNA: two clear peaks). Changing the amino acid residues at position 1 (Gln to Ala, DinB Q/PCNA), position 4 (Leu to Ala, DinB L/PCNA) and position 7 (Tyr to Ala, DinB Y/PCNA) also resulted in MacDinB-1 derivatives that failed to co-elute with MacPCNA in the gel filtration analysis. These results are portrayed by double peaks in the respective chromatograms (Fig. 10A), and also by a shift to later elution of proteins from the column (Fig. 10B, SDS-PAGE panels) compared to the experiments with wild type DinB-1 and PCNA. On the other hand, conversion of the residues to alanine at position 2 (Thr to Ala, DinB T/PCNA), position 3 (Ser to Ala, DinB S/PCNA), position 5 (Phe to Ala, DinB F/PCNA) and position 6 (Asp to Ala, DinB D/PCNA) did not show any visible effect on interaction with MacPCNA. 


\section{DISCUSSION}

The number of archaeal translesion DNA polymerases has increased rapidly in the publicly available databases due to more archaeal genomes being sequenced (Microbial Genome Database for Comparative Analysis: http://mbgd.genome.ad.jp/). However, only a few homologs have been biochemically characterized, and all of the reported proteins to date are from the Sulfolobales ${ }^{9 ; 18 ; 19 ; 31}$, a single genus in the archaeal subdomain Crenarchaeota. It is also important to note an important difference between the two subdomains of archaea. The crenarchaea have a heterotrimeric PCNA, while the euryarchaea have a homotrimeric PCNA as found in eukaryotes, such as the yeast and human ${ }^{46 ; 47}$. The Euryarchaeota constitutes a major subdomain in archaea, and most of the currently culturable archaea, including the methanogens, belong to this group. Furthermore, previous observation that two DinB proteins (Dpo4 and Dbh) from two members of the genus Sulfolobus exhibit important biochemical differences 9;20;31;39;40 suggest that there may be yet uncovered biochemical activities in other archaeal DinB homologs, especially those from different subdomains. Thus, the importance of characterizing family Y DNA polymerases or DinB homologs in the Euryarchaeota cannot be overlooked.

As shown in the phylogenetic tree (Fig. 1), available archaeal DinB proteins fall into five different groups. Also, in the present report, it is shown that archaea of the genus Methanosarcina generally contain two DinB homologs that group differently on the phylogenetic tree. For being the first DinB characterized out of the two homologs in the Methanosarcina spp, we have designated the protein in the current study DinB-1, implying that the other DinB homolog will be referred to as DinB-2. This report, 
therefore, examines for the first time the biochemical properties of a methanosarcinal DinB-1. Interestingly, the gene encoding DinB-2 is degenerated in the M. acetivorans genome (Genbank accession \# NP_615926), although in M. mazei, M. barkeri and Methanococcoides burtonii the gene is preserved. Confirmation of the degeneration of the gene encoding MacDinB-2 has been carried out in our lab through cloning and sequencing (Lin et al. unpublished).

The $M$. acetivorans DinB-1 can facilitate TLS through the lesion site of abasic DNA and CPD, and these activities are stimulated by the cognate sliding clamp through a PIP-box found at the C-terminus of MacDinB-1. On non-damaged DNA, the correct nucleotides were incorporated, and an "A" was preferably incorporated opposite an abasic lesion. The polymerase incorporated two A's upon encountering a cis-syn thymine-thymine dimer. The results presented in Fig. 5 also suggest that, in general, MacDinB-1 is able to discriminate between correct and incorrect nucleotides, and that the polymerase likely follows the ' $A$ ' rule when synthesizing through abasic lesions ${ }^{41}$. These properties are similar to those reported for Sulfolobus solfataricus P2 Dpo4, which was also observed to discriminate correct from incorrect nucleotides although some misincorporation was also observed ${ }^{31}$. Despite the observed error-free synthesis by MacDinB-1, the number of lesions tested in the present work is few, and there is a chance of later studies reporting lesions that render the polymerase error-prone. The MacDinB-1 on its own exhibits distributive DNA synthesis, and also appears to pause after incorporation of nucleotide upon encountering an abasic lesion or a cis-syn thyminethymine dimer. However, it can overcome the pause and longer products are synthesized in the presence of the cognate PCNA, perhaps due to increased affinity of the polymerase 
for the 3'-hydroxyl of the primer in the presence of PCNA, as described for the human DNA polymerase $\lambda^{42}$. It is also possible that PCNA increases the DNA polymerase's affinity for nucleotides as was reported earlier for some eukaryotic translesion DNA polymerases ${ }^{43 ; 44 ; 45}$. MacDinB-1 did not incorporate a base upon encountering a 6-4 photoproduct lesion, in contrast to $S$. solfataricus Dpo4 which was able to by-pass the (64) photoproduct lesion ${ }^{31}$.

We also observed an unusually long DNA synthesis by MacDinB-1 in vitro in the presence of MacPCNA as reported for M. acetivorans DNA polymerase BI under the same conditions ${ }^{21}$. The ortholog of MacDinB-1 in M. mazei also showed the same property in the presence of MacRFC and MacPCNA (results not shown). Therefore, from the sequence comparison, we assumed that the insertion sequence in motif III (Fig. 2), which harbors active site residues, plays a role in the unusually long products synthesized by the DinB-1 proteins. Deleting the six-amino acid insertion sequence (residue $101-106$, Fig. 2) in motif III of MacDinB-1, however, did not affect the biochemical properties compared to the wild type polymerase (results not shown). It is possible, however, that an effect of this deletion may be observed with other substrates in the future.

Unlike the DinB-1 polymerases, we postulate that the DinB-2 polymerases do not completely replicate the M13 phage genome $(7.2 \mathrm{~kb})$ in the presence of their cognate PCNA. We initially attempted to produce M. mazei DinB-2 in E. coli cells, however, due to insolubility problems with this protein, we purified the ortholog from Methanoculleus marisnigri, a member of Cluster V (Fig. 1). In the presence of its cognate PCNA (MmarisPCNA) or the heterologous MacPCNA and MacRFC, the M. marisnigri DinB 
made mostly $0.5 \mathrm{~kb}$ fragments (Supplementary Fig. 2), which are similar to the products made by the $S$. solfataricus $\mathrm{DinB}$ in the presence of its sliding clamp ${ }^{46}$. Therefore, the Methanosarcinales DinB-2 proteins and the other members of Cluster V may behave more like the Sulfolobales DinB in the presence of their sliding clamp.

Examination of the PIP-box shows that some residues are highly conserved (Fig. 8). We, therefore, investigated the contribution of the amino acid residues at various positions in the PIP-box of MacDinB-1 to its stimulation by MacPCNA during DNA synthesis. It was observed that mutating certain individual amino acid residues also led to dramatic decreases in the length of the product synthesized by the mutant MacDinB-1 proteins in the presence of MacPCNA. Furthermore, each of the mutations that led to a decrease in the size of product also resulted in lack of the capacity to co-elute with PCNA during gel filtration analysis. Thus, the basis for the reduced primer extension activity of these mutants is their impaired interaction with the sliding clamp. Although the gel filtration analysis suggested lack of interaction of the mutants with PCNA, the results, as shown in Fig. 9A and 9B, demonstrated that the mutants are stimulated by PCNA. However, due to weakened interactions with the sliding clamp, the mutant MacDinB-1 proteins are more prone to dissociation from the template, hence the ladder formation by products when resolved on acrylamide gel (Fig. 9B). Interestingly, two of the three critical residues whose mutations impair interaction with MacPCNA are highly conserved in several M. acetivorans PCNA-interacting proteins and also some proteins known to interact with the eukaryotic PCNA ${ }^{47}$.

The discovery of a TLS DNA polymerase that is capable of synthesizing very long DNA fragments in the presence of PCNA in vitro, is very significant, since such 
polymerases are thought to be mutagenic and hence cannot be permitted to synthesize long stretches of DNA in cells. We examined DNA synthesis by MacDinB-1 in the presence of the methanosarcinal replication protein A (RPA or ssDNA-binding proteins) homologs, MacRPA1, MacRPA2, and MacRPA3 ${ }^{48}$, which can coat exposed ssDNA templates during DNA synthesis in the cell. Also, since we do not currently know the cellular $\mathrm{pH}$ of M. acetivorans, we also tested the DNA synthesis of MacDinB-1 in the presence of PCNA and RFC at $\mathrm{pH}$ ranging from $\sim 6.0$ to $\sim 9.0$. Under the RPA and $\mathrm{pH}$ conditions, MacDinB-1 still synthesized long DNA products $(>3 \mathrm{~kb})$ with its cognate sliding clamp in vitro (Lin et al., unpublished), and this is six times the length reported for the $S$. solfatarcus homologs. We are cognizant of the fact that there might still be a factor or combination of factors in $M$. acetivorans cells that may repress the length of the products of PCNA-dependent DNA synthesis by MacDinB-1. Experiments are currently underway in our laboratory to test this hypothesis.

We did not show any evidence that MacDinB-1 actually functions in the $M$. acetivorans cells in this study as other reports about archaeal TLS polymerases. However practical genetic system to transform with exogenous genes and to knockout specific genes is available for this strain ${ }^{50 ; 51}$, and our further genetic research on this protein will contribute to the complete understanding of the in vivo functions of MacDinB-1. The current biochemical analysis of the first euryarchaeal DinB homolog sheds further light on the properties, potential, and diversity of members of the Y-family of DNA polymerases. 


\section{MATERIALS AND METHODS}

\section{Cloning of the genes encoding DinB homologs}

The gene (NP_618896) encoding a DinB-like translesion DNA polymerase in $M$. acetivorans (Mac) was amplified from the M. acetivorans genomic DNA with a pair of PCR primers named MacDinB-1-F and MacDinB-1-R, representing the forward and reverse primers, respectively (Table 1). The forward primer incorporated a NdeI site and the reverse primer incorporated a Sall site. After successful amplification of the approximately $1.1 \mathrm{~kb}$ fragment, the PCR product was cloned into a TA-cloning vector pGEMT (Promega), and the insert was sequenced (W. M. Keck Center for Functional and Comparative Genomics, University of Illinois at Urbana-Champaign) to verify the integrity of the coding sequence. The correct DNA insert was released by digestion with NdeI and SalI and ligated into a pET28a vector (Novagen) that had been predigested with NdeI and XhoI. The ligation placed the coding sequence in-frame with six histidines (His 6 -tag) encoded by the plasmid, and hence when expressed, the product carried an $\mathrm{N}$ terminal His 6 -tag to facilitate its purification. The cloning of MacPCNA and MacRFC were as described in our previous report ${ }^{21}$

\section{Site-directed mutagenesis and truncations in MacDinB-1}

A PCNA-interacting protein (PIP)-box-like sequence (QTSLFDY) at the extreme Cterminus of MacDinB-1 was deleted from the coding sequence by a PCR amplification that combined MacDinB-1 $\Delta \mathrm{C}-\mathrm{R}$ with MacDinB-1-F as the primer pair (Table 1). A six amino acid residue insertion (DPGENG, position 101 - 106) in M. acetivorans DinB (Fig. 2, motif III) was removed from the polypeptide by an initial PCR protocol that amplified 
the fragments upstream (amino acids position 1 - 100) and downstream (amino acid position 107 - 366) of the insertion sequence. The two PCR products, which contained overlapping sequences, were then fused into a single open reading frame through a third PCR amplification. The primer pair for the upstream fragment was MacDinB-1-F and MacDinB-1-Fu-R and that for the downstream fragment was MacDinB-1-Fu-F and MacDinB-1-R. The method used in making the fused fragment is described in detail in our previous reports ${ }^{22 ; 23}$. Each residue in the PIP box-like sequence (QTSLFDY) was mutated to alanine by use of a reverse primer that incorporated the necessary nucleotide changes directly into the oligonucleotides. This was possible since the PIP-box is at the extreme C-terminus of the protein. The template for mutagenic PCR amplification was the TA cloning vector (pGEMT) containing the MacDinB-1 wild type gene constructed as described above. Each reverse primer that was combined with the forward primer, MacDinB-1-F, for the site directed mutagenic PCR is presented in Table 1. After sequencing to confirm success in generating the targeted deletion or mutation, each fragment was cloned into pET28a (gene expression vector) as described above.

\section{Purification of recombinant proteins}

The pET28a vector used in the study contains an ampicillin resistance gene, instead of

the kanamycin resistance gene ${ }^{21}$. Thus, for gene expression, each construct was transformed into E. coli BL21-CodonPlus (DE3) RIL cells (Stratagene) by the heat shock method, and transformants were selected on LB plates containing ampicillin $(100 \mu \mathrm{g} / \mathrm{ml})$ and chloramphenicol $(50 \mu \mathrm{g} / \mathrm{ml})$. After an overnight incubation at $37^{\circ} \mathrm{C}$, a single colony from each plate was picked and cultured at $37{ }^{\circ} \mathrm{C}$ with vigorous shaking in LB broth 
containing the two antibiotics at the same concentrations as in the LB plates. When the absorbance at $600 \mathrm{~nm}$ reached 0.3 , gene expression was induced by addition of IPTG (isopropyl- $\beta$-D-thiogalactopyranoside) to a final concentration of $0.1 \mathrm{mM}$. The cells were further cultured at $16{ }^{\circ} \mathrm{C}$ for 16 hours followed by harvesting through centrifugation. The cell pellets were re-suspended in lysis buffer (50 mM sodium phosphate, $\mathrm{pH} 7.0,300 \mathrm{mM}$ $\mathrm{NaCl}$ ) and cell contents were released by use of a French pressure cell (Aminco). After removal of cell debris (centrifugation at $10,000 \mathrm{x}$ g for 20 min at $4{ }^{\circ} \mathrm{C}$ ), the supernatant was applied to a lysis buffer-equilibrated metal affinity resin (TALON ${ }^{\mathrm{TM}}$ cobalt affinity resin, Clontech). The proteins that bound to the resin were washed with 10 column volumes of lysis buffer followed by elution with a buffer composed of the lysis buffer containing $150 \mathrm{mM}$ imidazole. For further purification, the fractions were pooled and dialyzed against buffer A (50 mM Tris- $\mathrm{HCl}, \mathrm{pH} 8.0$, and $100 \mathrm{mM} \mathrm{NaCl})$. The dialysate was applied to an anion exchange column (HiTrap Q XL $5 \mathrm{ml}$; GE Healthcare) fitted to an FPLC (AKTA Explorer 10, GE Healthcare). After washing with ten column volumes of buffer $\mathrm{A}$, the bound proteins were eluted with a linear gradient $(0.1-1.0 \mathrm{M} \mathrm{NaCl})$ of buffer $\mathrm{B}(50 \mathrm{mM}$ TrisHCl, $\mathrm{pH} 8.0$, and $1.0 \mathrm{M} \mathrm{NaCl})$ at a flow rate of $1 \mathrm{ml} / \mathrm{min}$ at $4{ }^{\circ} \mathrm{C}$. Aliquots of eluted fractions were examined by $12 \%$ SDS-PAGE and highly purified proteins were dialyzed against a buffer containing $50 \mathrm{mM}$ Tris- $\mathrm{HCl}$, $\mathrm{pH} 8.0,100 \mathrm{mM}$ $\mathrm{NaCl}, 10 \%$ glycerol, and $0.5 \mathrm{mM}$ DTT for storage at $4{ }^{\circ} \mathrm{C}$. The purification methods for M. acetivorans PCNA (sliding clamp) and replication factor C (RFC) or clamp loader were the same as earlier reported ${ }^{21}$.

\section{Gel filtration chromatography}


Purified proteins, either as individual proteins or mixed with PCNA, were dialyzed against a buffer containing $50 \mathrm{mM}$ sodium phosphate, $\mathrm{pH} 7.0$, and $150 \mathrm{mM} \mathrm{NaCl}$. The dialysates were applied to a Superdex 200 HR 10/30 column (GE Healthcare) preequilibrated with the same buffer. In each case, the chromatography was developed with the same buffer at a flow rate of $0.5 \mathrm{ml} / \mathrm{min}$. Fractions of volume $0.5 \mathrm{ml}$ were collected and aliquots were resolved by $12 \%$ SDS-PAGE. To determine whether MacDinB-1 and MacPCNA interact in solution, equal amounts of each protein $(20 \mathrm{pmol} / \mu \mathrm{l})$ were mixed and dialyzed against the dialysis buffer (50 mM sodium phosphate, $\mathrm{pH} 7.0$, and $150 \mathrm{mM}$ $\mathrm{NaCl})$ at $4{ }^{\circ} \mathrm{C}$ overnight. The mixture was then analyzed by gel filtration as described above for the individual proteins. Where mutants of MacDinB-1 were investigated for interaction with MacPCNA, the respective mutant replaced the wild type MacDinB-1 during dialysis prior to gel filtration analysis. Aliquots from fractions collected for each gel filtration analysis were examined through $12 \%$ SDS-PAGE. The size exclusion chromatography column was calibrated by running a set of protein standards (ferritin: $440 \mathrm{kDa}$, catalase: $232 \mathrm{kDa}$, aldolase: $158 \mathrm{kDa}$, albumin: $67 \mathrm{kDa}$, ovalbumin: $43 \mathrm{kDa}$, and ribonuclease A: $13.7 \mathrm{kDa}, \mathrm{GE}$ Healthcare) under the same conditions as used for the proteins under investigation.

\section{Translesion DNA synthesis on damaged DNA}

Translesion DNA synthesis was performed with a $5^{\prime}$-labeled $\left[\gamma^{-32} \mathrm{P}\right]$ 27-mer oligonucleotide annealed to a linear template of size 49 nucleotides 5'd(AGCTACCATGCCTGCACGAATTAAGCAATTCGTAATCATGGTCATAGCT)-3' .

The non-damaged DNA substrate harbored no lesion in the template, and three different 
lesion-containing templates were used. The lesions were a synthetic abasic site (abasic DNA), a cis-syn cyclobutane pyrimidine thymine-thymine dimer (CPD), and (6-4) photoproduct. The (6-4) photoproduct and the cis-syn thymine-thymine dimer were located at the $21^{\text {st }}-22^{\text {nd }}$ nucleotides from the 5 ' terminus of the template, and the oligonucleotides were synthesized as described previously ${ }^{24 ; 25}$. The abasic site was at position 22 from the 5 ' terminus of the template DNA and was synthesized as described by Fujiwara and co-workers ${ }^{26}$. Translesion DNA synthesis was carried out in a buffer composed of $20 \mathrm{mM}$ Tris- $\mathrm{HCl}(\mathrm{pH}$ 8.8), $2 \mathrm{mM} \beta$-mercaptoethanol, $5 \mathrm{mM} \mathrm{MgCl} 2,100$ $\mu \mathrm{g} / \mathrm{ml}$ bovine serum albumin (BSA), $5 \mathrm{nM}$ DNA, $250 \mathrm{nM}$ dNTP and $500 \mathrm{nM}$ MacDinB-1. Where the effect of PCNA was examined, the experiment was carried out at $\mathrm{pH} 6.5$ because the physical interaction of MacDinB-1 and MacPCNA is tighter at $\mathrm{pH} 6.5$ as compared to $\mathrm{pH} 8.8$ from our Surface Plasmon Resonance analyses (Lin et al. unpublished).

\section{Primer extension analysis on long DNA (M13mp18 ssDNA: 7.2 kb) template}

To create the substrate for primer extension analysis using a long DNA template, 2 pmol of an oligonucleotide $5^{\prime}$-end-labeled with $\left[\gamma^{-}{ }^{32} \mathrm{P}\right]$ was annealed to $1.0 \mu \mathrm{g}$ of M13mp18 circular ssDNA by heating in DNA polymerase buffer $(20 \mathrm{mM}$ Tris- $\mathrm{HCl}, \mathrm{pH} 8.8,100$ $\mathrm{mM} \mathrm{NaCl}, 5 \mathrm{mM} \mathrm{MgCl}$, and $1 \mathrm{mM} \beta$-mercaptoethanol) to $95{ }^{\circ} \mathrm{C}$ for $5 \mathrm{~min}$ and then gently cooled to room temperature. The oligonucleotide is based on positions 6205 to 6234 of the M13mp18 phage genome ${ }^{27}$. The standard reaction mixture $(20 \mu \mathrm{l})$ was composed of the labeled substrate in $20 \mathrm{mM}$ Tris- $\mathrm{HCl}$, pH $8.8,100 \mathrm{mM} \mathrm{NaCl}, 5 \mathrm{mM}$ $\mathrm{MgCl}_{2}, 2 \mathrm{mM} \beta$-mercaptoethanol, and $250 \mu \mathrm{M}$ of each dNTP, and to initiate the reaction, 
MacDinB-1 was added at $20 \mathrm{pmol} / \mathrm{reaction}$. Where effects of accessory proteins (MacPCNA and MacRFC) were investigated, ATP was added at $1 \mathrm{mM}$ to the reaction mixture and MacPCNA and MacRFC were added at $0.3 \mu \mathrm{M}$ (based on PCNA trimer) and

$90 \mathrm{nM}$, respectively, per reaction ${ }^{21}$. In the experiments that investigated the mutant forms of MacDinB-1 protein, each reaction mixture was the same as that for wild type MacDinB-1, except for replacement of MacDinB-1 with the mutant under investigation. The reaction mixtures were incubated at $37{ }^{\circ} \mathrm{C}$ for $30 \mathrm{~min}$ and each reaction was terminated by adding $6 \mu \mathrm{l}$ of stop solution (98\% formamide, $1 \mathrm{mM}$ EDTA, $0.1 \%$ xylene cyanol, $0.1 \%$ bromphenol blue). After heating at $95{ }^{\circ} \mathrm{C}$ for $5 \mathrm{~min}$, the products were resolved on $1 \%$ alkali agarose gel in $50 \mathrm{mM}$ sodium hydroxide and $1 \mathrm{mM}$ EDTA or on $8 \%$ acrylamide gel containing $8 \mathrm{M}$ urea. Visualization of the products was through autoradiography.

\section{Phylogenetic analysis of archaeal family $Y$ polymerases}

Thirty archaeal DinB protein sequences were retrieved from the Genbank (http://www.ncbi.nlm.nih.gov/) and used to study the phylogeny of archaeal DinB homologs. The sequences were aligned using the multiple sequence alignment software Clustal X ver.1.83 ${ }^{28}$. The polypeptide sequence alignment was then used to generate a phylogenetic tree based on the neighbor-joining method ${ }^{29}$. An unrooted tree was drawn from the calculated tree using the unrooted tree program provided with the NJplot program ${ }^{30}$. The organisms from which the polypeptides originated and the Genbank accession numbers of each protein sequence were as follows: M. acetivorans (Mac) DinB (Accession: NP_618896), Methanosarcina mazei (Mma) DinB-1 (Accession: 
NP_632910), Methanosarcina barkeri (Mba) DinB-1 (Accession: YP_304121), Methanococcoides burtonii (Mbu) DinB-1 (Accession:YP_566785), Methanosarcina barkeri DinB-2 (Accession: YP_306247), Methanococcoides burtonii DinB-2 (Accession:YP_564911), Methanosarcina mazei DinB-2 (Accession: NP_634900), Sulfolobus solfataricus P1 (Sso) Dbh (Accession: AAB38090), Sulfolobus solfataricus P2 Dpo4 (Accession: NP_343798), Sulfolobus acidocaldarius (Sac) Dbh (Accession: YP_255242), Nitrosopumilus maritimus (Nma) DinB (Accession: YP_001581976), Cenarchaeum symbiosum (Csym) DinB (Accession: ABK77022), Methanoculleus marisnigri (Mmaris) DinB (Accession: YP_001046295), Stygiolobus azoricus (Saz) Dbh (Accession: ABA03147), Sulfolobus tokodaii (Sto) Dbh (Accession: NP_376460), Sulfurisphaera ohwakuensis (Soh) Dbh (Accession: ABA03148), Acidianus infernus (Ain) Dpo4 (Accession: ABA03146), Methanospirillum hungatei (Mhu) DinB (Accession: YP_502922), Candidatus Methanoregula boonei (Mbo) DinB (Accession: YP_001403783), Sulfolobus tokodaii str.7 (sto7) Dbh (Accession: NP_376460), Sulfolobus shibatae (Ssh) Dpo4 (Accession: ABA03149), Halobacterium species NRC-1 (Hsp NRC-1) DinB (Accession: NP_279949), Picrophilus torridus (Pto) DinB (Accession: YP_023933), Ferroplasma acidarmanus (Fac) DinB (Accession: ZP_01709751), Halobacterium salinarium (Hsa) Dpo4 (Accession: Q9HQT4), Natronomonas pharaonis (Npha) DinB (Accession: YP_327108), Halorubrum lacusprofundi (Hla) DinB (Accession: ZP_02014687), Haloarcula marismortui (Hma) DinB (Accession: YP_135264), Haloquadratum walsbyi (Hwa) DinB (Accession: YP_659045), Metallosphaera sedula (Mse) Dpo4 (Accession: YP_001190697). 


\title{
FUNDING
}

This research was supported by a National Science Foundation Grant (MCB 0238451) to IKOC.

\section{ACKNOWLEDGEMENTS}

We thank Dr. William Metcalf, University of Illinois at Urbana-Champaign (UIUC) for providing M. acetivorans genomic DNA.

\begin{abstract}
ABBREVIATIONS
Pol-DNA polymerase, CPD- cis-syn cyclobutane pyrimidine thymine-thymine dimer, BSA,-bovine serum albumin, ATP-Adenosine triphsophate, dNTP-deoxyribonucleotide triphosphate, SDS-PAGE-sodium dodecyl sulfate polyacrylamide gel electrophoresis, EDTA-ethylenediaminetetraacetic acid, PCNA-proliferating cell nuclear antigen, RFCreplication factor C, RPA-replication protein A, IPTG- isopropyl- $\beta$-Dthiogalactopyranoside.
\end{abstract}




\section{TABLES}

Table 1: Oligonucleotides used in this study

\begin{tabular}{|c|c|c|}
\hline Experiment & Oligonucleotide & Nucleotide sequence \\
\hline \multirow[t]{2}{*}{ MacDinB-1 WT } & MacDinB-1-F & 5' CATATGATGCAGCGGGTAATTATTCATGTAG 3' \\
\hline & MacDinB-1-R & 5' GTCGACTTAATAATCAAAAAGGCTTGTCTGACC 3' \\
\hline \multirow[t]{3}{*}{ MacDinB-1 truncation } & MacDinB-1 $\Delta \mathrm{C}-\mathrm{R}$ & 5' GTCGACTTAACCTTTTTTTTTTCTGAAGCCCCCCTAC 3' \\
\hline & MacDinB-1-Fu-R & 5' AACACTTATCTGTTCAAAGGAATCTGCATAGCTGCGAAGGATTTCCAT 3' \\
\hline & MacDinB-1-Fu-F & 5' ATGGAAATCCTTCGCAGCTATGCAGATTCCTTTGAACAGATAAGTGTT 3' \\
\hline \multirow[t]{7}{*}{$\begin{array}{l}\text { MacDinB-1 } \\
\text { mutagenesis }\end{array}$} & $\begin{array}{l}\text { MacDinB-1 } \\
\text { Q360A-R }\end{array}$ & 5' GTCGACTTAATAATCAAAAAGGCTTGTCGCACCTTTTTT 3' \\
\hline & $\begin{array}{l}\text { MacDinB-1 } \\
\text { T361A-R }\end{array}$ & 5' GTCGACTTAATAATCAAAAAGGCTTGCCTGACCTTTTTTT 3' \\
\hline & $\begin{array}{l}\text { MacDinB-1 } \\
\text { S362A-R }\end{array}$ & 5' GTCGACTTAATAATCAAAAAGGGCTGTCTGACC 3' \\
\hline & $\begin{array}{l}\text { MacDinB-1 } \\
\text { L363A-R }\end{array}$ & 5' GTCGACTTAATAATCAAAAGCGCTTGTCTGACC 3' \\
\hline & $\begin{array}{l}\text { MacDinB-1 } \\
\text { F364A-R }\end{array}$ & 5' GTCGACTTAATAATCAGCAAGGCTTGTCTGACC 3' \\
\hline & $\begin{array}{l}\text { MacDinB-1 } \\
\text { D365A-R }\end{array}$ & 5' GTCGACTTAATAAGCAAAAAGGCTTGTCTGACC 3' \\
\hline & $\begin{array}{l}\text { MacDinB-1 } \\
\text { Y366A-R }\end{array}$ & 5' GTCGACTTAAGCATCAAAAAGGCTTGTCTGACC 3' \\
\hline Primer extension & M13 6205-6234 & 5' ATTCGTAATCATGGTCATAGCTGTTTCCTG 3' \\
\hline
\end{tabular}

The oligonucleotides listed for MacDinB-1 WT were used for amplification of the MacDinB-1 gene from the genomic DNA. The oligonucleotides listed for MacDinB-1 truncation were combined with the MacDinB-1 WT oligonucleotides to delete a six amino acid-residue insertion sequence (Fig. 2, DPGENG in motif III), and to delete the PIP-box at the extreme C-terminus of MacDinB-1 (Fig. 2, motif VI). The oligonucelotides listed for MacDinB-1 mutagenesis contained the nucleotide changes that introduced mutations in the MacDinB-1 PIP-box. As an example, a combination of the forward primer MacDinB-1-F and MacDinB-1 Q360A R in PCR with the MacDinB-1 gene as template led to a mutant MacDinB-1 with a Q360A mutation. The oligonucleotide listed for primer extension was labeled with ${ }^{32} \mathrm{P}$ and annealed as the primer to M13mp18 ssDNA (template) for primer extension experiments. 


\section{FIGURE LEGENDS}

Fig 1. An unrooted phylogenetic tree of DinB homologs in archaea showing five different clusters. Thirty archaeal DinB protein sequences were retrieved from the protein database at the Genbank. The sequences were aligned with Clustal $\mathrm{X}$ and the alignment was used in generating a phylogenetic tree based on the neighbor joining method. For detailed description and full names of archaeal organisms and accession number of proteins see Materials and Methods. MacDinB-1, which was biochemically characterized in this report is in cluster II and is marked with an asterisk. Bar, 0.2 substitutions per amino acid position.

Fig. 2. An alignment of 30 archaeal DinB homologs showing six major conserved

motifs. The conserved motifs I to $\mathrm{V}$ were defined by Kulaeva et al. ${ }^{6}$. The amino acid residues that are identical (black) or similar (gray) at $>50 \%$ of the positions are indicated. The active site sequence for most bypass polymerases, $\mathrm{S}(\mathrm{I} / \mathrm{L} / \mathrm{V}) \mathrm{DE}$, is in motif III. The asterisks indicate residues necessary for catalysis ${ }^{39}$. Motif VI is located at the C terminal region and represents a PCNA-interacting protein-box, QXXhXXaa, defined as: $\mathrm{h}$, residues with moderately hydrophobic side chain; a, residues with highly hydrophobic, aromatic side chain; $X$, any residue. The glutamine (Q) at position 1 is not conserved in the crenarchaeal DinB proteins. The organisms in which the first position is neither glutamine (Q) nor asparagine $(\mathrm{N})$ belong to the Crenarchaeota, which have a heterotrimeric PCNA, whereas the others, except for NmaDinB and CsymDinB, are Euryarchaeota and have a homotrimeric PCNA as in eukaryotes. The NmaDinB and CsymDinB are from N. maritimus and C. symbiosum, which are members of the newly 
proposed archaeal sudomain Thaumarchaaeota ${ }^{36}$. For a key to the abbreviation of organism names, refer to the Materials and Methods section.

Fig. 3. Purification of M. acetivorans DinB-1 and its accessory proteins. Molecular mass markers (lane 1), MacDinB-1 (lane 2), MacDinB-1 $\Delta \mathrm{T}$, a protein with motif VI (Fig. 2) deleted (lane 3), MacPCNA or sliding clamp (lane 4), and MacRFC or clamp loader (lane 5). The proteins were purified as described in the Materials and Methods, and $1 \mu \mathrm{g}$ of protein/lane were resolved on $12 \%$ sodium dodecyl sulfate-polyacrylamide gel electrophoresis (SDS-PAGE) and stained with Coomassie brilliant blue G250 for visualization.

Fig. 4. Translesion DNA synthesis by MacDinB-1. A. A schematic representation of template DNA in the following order: non-damaged DNA, abasic DNA (Ab), cyclobutane pyrimidine dimer (CPD), and (6-4) photoproduct (6-4). The asterisks show the radioisotopic $\left({ }^{32} \mathrm{P}\right)$ labeling. B. DNA synthesis through non-damaged DNA at increasing concentrations (as indicated) of MacDinB-1. The primer extension reaction was carried out in a buffer described in Materials and Methods. The primer and the template lengths were 27 -mer and 49-mer, respectively, as indicated in A. The reactions were carried out at $37{ }^{\circ} \mathrm{C}$ for $10 \mathrm{~min}$ and the products were denatured by boiling and resolved on a $10 \%$ acrylamide gel containing $8 \mathrm{M}$ urea. The extent of primer utilization is given below each lane as $\%$ by quantification of the band intensities using the software Multi Gauge ver. 3 (Fiji film), with the band intensity of the primer in the most left lane as $0 \%$. C. DNA synthesis at MacDinB- 1 concentration of $0.5 \mu \mathrm{M}$ with non-damaged 
DNA, abasic DNA (Ab), cyclobutane pyrimidine dimer (CPD), and 6-4 photoproduct (64) as substrates. The reactions were carried out at $37^{\circ} \mathrm{C}$ for the indicated times. The extent of primer utilization, as obtained by the same procedure as in $\mathrm{B}$, is given below each lane.

Fig. 5. Preference of nucleotide incorporation by MacDinB-1. The extent of incorporation was measured with DNA templates without damage (A), containing abasic site (B), and CPD (C), in the absence (labeled 0) or presence of all four dNTP's (labeled $4 n$ ) or presence of each individual dNTP (labeled G, A, T, C). The substrates were the same as described in Fig. 4A, however, only the region around the lesion is shown underneath each gel. The primer extension reaction was carried out in a buffer described in Materials and Methods at $37{ }^{\circ} \mathrm{C}$ for $10 \mathrm{~min}$. The enzyme concentrations were $0.1 \mu \mathrm{M}$ for non-damaged DNA and $1 \mu \mathrm{M}$ for $\mathrm{Ab}$ and $\mathrm{CPD}$, respectively. The products were denatured by boiling and resolved on a $10 \%$ acrylamide gel containing $8 \mathrm{M}$ urea. In each panel, it is observed that addition of all 4 nucleotides ( $4 n$ ) to the reaction mixture leads to synthesis of larger products. A. The correct nucleotide "A" was incorporated opposite the templating nucleotide "T" in a non-damaged DNA template in the panel A. Incorporation of "A" with the templating nucleotide being an abasic lesion was observed in the panel B. Incorporation of "A's" with the templating nucleotide being a CPD (TT) lesion was observed in the panel C.

Fig. 6. MacPCNA stimulates translesion DNA synthesis by MacDinB-1. The primer extension reactions were carried out at a constant MacDinB-1 concentration $(0.5 \mu \mathrm{M})$ in 
the presence of MacPCNA (the concentrations were given above each lane) using the template containing abasic DNA (A) and CPD (B) lesions at $37^{\circ} \mathrm{C}$ for $10 \mathrm{~min}$. Increased DNA synthesis through an abasic DNA lesion (A), and a CPD lesion (B) were observed with increasing concentrations of MacPCNA. The $\mathrm{pH}$ of the reaction buffer in this experiment was 6.5, instead of 8.8 as used in Fig. 4 and Fig. 5, to enhance the effect of PCNA on the reaction, because the physical interaction of MacDinB-1 and MacPCNA is more tight at $\mathrm{pH} 6.5$ as compared to $\mathrm{pH} 8.8$ from our Surface Plasmon Resonance analyses (data not shown). The template in each experiment was a short linear DNA as described in Fig. 4A, and therefore a clamp loader was not required to load PCNA onto the DNA. The lane marked $\mathrm{P}$ is the substrate alone (migration of ${ }^{32} \mathrm{P}$-labeled-primer) without any protein. The rest of the lanes received MacDinB-1 $(0.5 \mu \mathrm{M})$ and the indicated amounts of PCNA. The products were denatured by boiling and resolved on an $8 \%$ acrylamide gel containing $8 \mathrm{M}$ urea.

Fig. 7. MacDinB-1 and MacPCNA interaction. A. Elution profiles of gel filtration chromatographies of (i) MacDinB-1, (ii) MacPCNA, (iii) MacDinB-1/MacPCNA dialyzate, and (iv) MacDinB-1 $\Delta \mathrm{T} / \mathrm{MacPCNA}$ dialyzate were monitored at an absorbance of $280 \mathrm{~nm}$. MacDinB-1 $\Delta \mathrm{T}$ has the PCNA interacting protein (PIP)-box deleted. Each experiment was carried out three times. The peak elution volumes are the mean \pm standard deviations. B. SDS-PAGE analysis of fractions obtained from gel filtration chromatographies of MacDinB-1, MacPCNA, MacDinB-1/MacPCNA, and MacDinB1 $\Delta \mathrm{T} / \mathrm{MacPCNA}$. Aliquots from each fraction (elution volume from 11.0 to $16.5 \mathrm{ml}$ ) were 
analyzed by $12 \%$ SDS-PAGE and visualized by staining with Coomassie brilliant blue G250.

Fig. 8. PCNA-Interacting protein (PIP)-boxes in representative $M$. acetivorans proteins and some human proteins. Representatives of characterized and uncharacterized (putative) PIP-boxes in some $M$. acetivorans and two human proteins. The residues in positions 1, 4, and 7 are highly conserved (also refer to Fig. 2). PolB-I is a family B DNA polymerase containing two characterized PIP boxes ${ }^{21}$, RFCL is the twoPIP-box-containing replication factor $\mathrm{C}$ large subunit of $M$. acetivorans (uncharacterized); FEN-I is the Flap Endonuclease I of $M$. acetivorans (characterized PIP box, Lin et al. unpublished), Hjm is a Holliday junction unwinding helicase of $M$. acetivorans (Lin et al., unpublished); DP2 is the large subunit of the M. acetivorans DNA polymerase D (PolD) (uncharacterized), human FEN-I ${ }^{49}$, and $\mathrm{p} 21^{\mathrm{WAF} 1 / \mathrm{CIP} 1}$ is a cyclin dependent kinase inhibitor ${ }^{47}$. In the consensus sequence ' $\mathrm{x}$ ' is any amino acid.

Fig. 9. The primer extension of MacDinB-1 and individual mutants in the presence and absence of processivity factors (MacPCNA and MacRFC). Two pmol of a ${ }^{32} \mathrm{P}-$ labeled oligonucleotide annealed to $1 \mu \mathrm{g}$ of M13mp18 ssDNA was incubated with MacDinB-1 or the individual mutants in the presence of MacPCNA and MacRFC complex in a DNA polymerase reaction buffer as described in the Materials and Methods. The products of the primer extension reaction were resolved on (A) $1 \%$ alkaline agarose gel, and (B) denaturing (8M urea) $8 \%$ acrylamide gel electrophoresis. (WT: wild-type DinB; $\Delta$ : DinB with the PIP-box (QTSLFDY) cleaved; Alanine scanning of the PIP-box: 
$\mathrm{Q} \rightarrow \mathrm{A} ; \mathrm{T} \rightarrow \mathrm{A} ; \mathrm{S} \rightarrow \mathrm{A} ; \mathrm{L} \rightarrow \mathrm{A} ; \mathrm{F} \rightarrow \mathrm{A} ; \mathrm{D} \rightarrow \mathrm{A} ; \mathrm{Y} \rightarrow \mathrm{A}$. (C) In the absence of both accessory factors, MacDinB-1 was distributive and synthesize shorter fragments. The products of the primer extension reactions were denatured by boiling, resolved by denaturing ( $8 \mathrm{M}$ urea) $8 \%$ acrylamide gel electrophoresis, and then visualized by autoradiography.

Fig. 10. In vitro interactions of MacPCNA with wild type MacDinB-1 and MacDinB1 mutants with mutations in the PIP-box. (A) Chromatograms showing the elution profiles of MacDinB-1 wild type or its mutants dialyzed together with MacPCNA. The elution profiles from gel filtration were monitored at an absorbance of $280 \mathrm{~nm}$. (B) SDSPAGE analysis of aliquots of protein eluted from the gel filtration chromatography. Each fraction (elution volume from 11.0 to $16.5 \mathrm{ml}$ ) was analyzed with 12\% SDS-PAGE gels and stained with Coomassie brilliant blue G-250 for visualization. Also, shown under the mutation column are the various PIP-box mutants of MacDinB-1 dialyzed together with MacPCNA. In the interaction column, the plus and minus signs indicate detection of interaction and no interaction, respectively. The definitions for the abbreviations are as follows: DinB $\Delta$, MacDinB-1 with the PIP-box deleted; DinB Q, Q360A mutant; DinB T, T361A mutant; DinB S, S362A mutant; DinB L, L363A mutant; DinB F, F364A mutant; DinB D, D365A mutant; and DinB Y, Y366A mutant. 


\section{LITERATURE CITED}

1. Tang, M., Pham, P., Shen, X., Taylor, J. S., O'Donnell, M., Woodgate, R. \& Goodman, M. F. (2000). Roles of E. coli DNA polymerases IV and V in lesiontargeted and untargeted SOS mutagenesis. Nature 404, 1014-1018.

2. Indiani, C., McInerney, P., Georgescu, R., Goodman, M. F. \& O'Donnell, M. (2005). A sliding-clamp toolbelt binds high- and low-fidelity DNA polymerases simultaneously. Mol Cell 19, 805-815.

3. Johnson, R. E., Prakash, L. \& Prakash, S. (2005). Distinct mechanisms of cis-syn thymine dimer bypass by Dpo4 and DNA polymerase eta. Proc Natl Acad Sci US A 102, 12359-12364.

4. Ohmori, H., Friedberg, E. C., Fuchs, R. P., Goodman, M. F., Hanaoka, F., Hinkle, D., Kunkel, T. A., Lawrence, C. W., Livneh, Z., Nohmi, T., Prakash, L., Prakash, S., Todo, T., Walker, G. C., Wang, Z. \& Woodgate, R. (2001). The Y-family of DNA polymerases. Mol Cell 8, 7-8.

5. Tang, M., Shen, X., Frank, E. G., O'Donnell, M., Woodgate, R. \& Goodman, M. F. (1999). UmuD'(2)C is an error-prone DNA polymerase, Escherichia coli pol V. Proc Natl Acad Sci U S A 96, 8919-8924.

6. Kulaeva, O. I., Koonin, E. V., McDonald, J. P., Randall, S. K., Rabinovich, N., Connaughton, J. F., Levine, A. S. \& Woodgate, R. (1996). Identification of a $\mathrm{DinB} / \mathrm{UmuC}$ homolog in the archeon Sulfolobus solfataricus. Mutat Res 357, 245253.

7. Friedberg, E. C. \& Gerlach, V. L. (1999). Novel DNA polymerases offer clues to the molecular basis of mutagenesis. Cell 98, 413-416.

8. Fujii, S. \& Fuchs, R. P. (2004). Defining the position of the switches between replicative and bypass DNA polymerases. EMBO J 23, 4342-4352.

9. $\quad$ Gruz, P., Pisani, F. M., Shimizu, M., Yamada, M., Hayashi, I., Morikawa, K. \& Nohmi, T. (2001). Synthetic activity of Sso DNA polymerase Y1, an archaeal DinB-like DNA polymerase, is stimulated by processivity factors proliferating cell nuclear antigen and replication factor C. J Biol Chem 276, 47394-47401.

10. Haracska, L., Kondratick, C. M., Unk, I., Prakash, S. \& Prakash, L. (2001). Interaction with PCNA is essential for yeast DNA polymerase eta function. $\mathrm{Mol}$ Cell 8, 407-415.

11. Reuven, N. B., Arad, G., Maor-Shoshani, A. \& Livneh, Z. (1999). The mutagenesis protein UmuC is a DNA polymerase activated by UmuD', RecA, and SSB and is specialized for translesion replication. $J$ Biol Chem 274, 31763-31766.

12. Becherel, O. J., Fuchs, R. P. \& Wagner, J. (2002). Pivotal role of the beta-clamp in translesion DNA synthesis and mutagenesis in E. coli cells. DNA Repair (Amst) 1, 703-708.

13. Napolitano, R., Janel-Bintz, R., Wagner, J. \& Fuchs, R. P. (2000). All three SOSinducible DNA polymerases (Pol II, Pol IV and Pol V) are involved in induced mutagenesis. EMBO J 19, 6259-6265.

14. Lehmann, A. R. (2006). New functions for Y family polymerases. Mol Cell 24, 493-495. 
15. Jarosz, D. F., Godoy, V. G. \& Walker, G. C. (2007). Proficient and accurate bypass of persistent DNA lesions by DinB DNA polymerases. Cell Cycle 6, 817822.

16. Tissier, A., Frank, E. G., McDonald, J. P., Iwai, S., Hanaoka, F. \& Woodgate, R. (2000). Misinsertion and bypass of thymine-thymine dimers by human DNA polymerase iota. $E M B O J \mathbf{1 9}, 5259-5266$.

17. Nelson, J. R., Lawrence, C. W. \& Hinkle, D. C. (1996). Deoxycytidyl transferase activity of yeast REV1 protein. Nature 382, 729-731.

18. Ling, H., Boudsocq, F., Woodgate, R. \& Yang, W. (2001). Crystal structure of a Y-family DNA polymerase in action: a mechanism for error-prone and lesionbypass replication. Cell 107, 91-102.

19. Ling, H., Boudsocq, F., Woodgate, R. \& Yang, W. (2004). Snapshots of replication through an abasic lesion; structural basis for base substitutions and frameshifts. Mol Cell 13, 751-762.

20. McDonald, J. P., Hall, A., Gasparutto, D., Cadet, J., Ballantyne, J. \& Woodgate, R. (2006). Novel thermostable Y-family polymerases: applications for the PCR amplification of damaged or ancient DNAs. Nucleic Acids Res 34, 1102-1111.

21. Chen, Y. H., Kocherginskaya, S. A., Lin, Y., Sriratana, B., Lagunas, A. M., Robbins, J. B., Mackie, R. I. \& Cann, I. K. (2005). Biochemical and mutational analyses of a unique clamp loader complex in the archaeon Methanosarcina acetivorans. J Biol Chem 280, 41852-41863.

22. Lin, Y., Guzman, C. E., McKinney, M. C., Nair, S. K., Ha, T. \& Cann, I. K. (2006). Methanosarcina acetivorans flap endonuclease 1 activity is inhibited by a cognate single-stranded-DNA-binding protein. J Bacteriol 188, 6153-6167.

23. Robbins, J. B., McKinney, M. C., Guzman, C. E., Sriratana, B., Fitz-Gibbon, S., Ha, T. \& Cann, I. K. (2005). The euryarchaeota, nature's medium for engineering of single-stranded DNA-binding proteins. J Biol Chem 280, 15325-15339.

24. Murata, T., Iwai, S. \& Ohtsuka, E. (1990). Synthesis and characterization of a substrate for $\mathrm{T} 4$ endonuclease $\mathrm{V}$ containing a phosphorodithioate linkage at the thymine dimer site. Nucleic Acids Res 18, 7279-7286.

25. Iwai, S., M. Shimizu, H. Kamiya, and E. Ohtsuka. (1996). Synthesis of a phosphoramidite coupling unit of the pyrimidine (6-4) pyrimidone photoproduct and its incorporation into oligodeoxynucleotides. J. Am. Chem. Soc. 118, 76427643.

26. Fujiwara, Y., Masutani, C., Mizukoshi, T., Kondo, J., Hanaoka, F. \& Iwai, S. (1999). Characterization of DNA recognition by the human UV-damaged DNAbinding protein. $J$ Biol Chem 274, 20027-20033.

27. Yanisch-Perron, C., Vieira, J. \& Messing, J. (1985). Improved M13 phage cloning vectors and host strains: nucleotide sequences of the M13mp18 and pUC19 vectors. Gene 33, 103-119.

28. Jeanmougin, F., Thompson, J. D., Gouy, M., Higgins, D. G. \& Gibson, T. J. (1998). Multiple sequence alignment with Clustal X. Trends Biochem Sci 23, 403405.

29. Saitou, N. \& Nei, M. (1987). The neighbor-joining method: a new method for reconstructing phylogenetic trees. Mol Biol Evol 4, 406-425. 
30. Perriere, G. \& Gouy, M. (1996). WWW-query: an on-line retrieval system for biological sequence banks. Biochimie 78, 364-369.

31. Boudsocq, F., Iwai, S., Hanaoka, F. \& Woodgate, R. (2001). Sulfolobus solfataricus P2 DNA polymerase IV (Dpo4): an archaeal DinB-like DNA polymerase with lesion-bypass properties akin to eukaryotic poleta. Nucleic Acids Res 29, 4607-4616.

32. Konneke, M., Bernhard, A. E., de la Torre, J. R., Walker, C. B., Waterbury, J. B. \& Stahl, D. A. (2005). Isolation of an autotrophic ammonia-oxidizing marine archaeon. Nature 437, 543-546.

33. Cann, I. K., Ishino, S., Hayashi, I., Komori, K., Toh, H., Morikawa, K. \& Ishino, Y. (1999). Functional interactions of a homolog of proliferating cell nuclear antigen with DNA polymerases in Archaea. J Bacteriol 181, 6591-6599.

34. Cann, I. K., Komori, K., Toh, H., Kanai, S. \& Ishino, Y. (1998). A heterodimeric DNA polymerase: evidence that members of Euryarchaeota possess a distinct DNA polymerase. Proc Natl Acad Sci U S A 95, 14250-14255.

35. Cann, I. K. \& Ishino, Y. (1999). Archaeal DNA replication: identifying the pieces to solve a puzzle. Genetics 152, 1249-1267.

36. Brochier-Armanet, C., Boussau, B., Gribaldo, S. \& Forterre, P. (2008). Mesophilic Crenarchaeota: proposal for a third archaeal phylum, the Thaumarchaeota. Nat Rev Microbiol 6, 245-252.

37. Angelov, A. \& Liebl, W. (2006). Insights into extreme thermoacidophily based on genome analysis of Picrophilus torridus and other thermoacidophilic archaea. $J$ Biotechnol 126, 3-10.

38. Warbrick, E. (1998). PCNA binding through a conserved motif. Bioessays 20, 195-199.

39. Zhou, B. L., Pata, J. D. \& Steitz, T. A. (2001). Crystal structure of a DinB lesion bypass DNA polymerase catalytic fragment reveals a classic polymerase catalytic domain. Mol Cell 8, 427-437.

40. Potapova, O., Grindley, N. D. \& Joyce, C. M. (2002). The mutational specificity of the Dbh lesion bypass polymerase and its implications. J Biol Chem 277, 28157-28166.

41. Strauss, B. S. (1991). The 'A rule' of mutagen specificity: a consequence of DNA polymerase bypass of non-instructional lesions? Bioessays 13, 79-84.

42. Maga, G., Villani, G., Ramadan, K., Shevelev, I., Tanguy Le Gac, N., Blanco, L., Blanca, G., Spadari, S. \& Hubscher, U. (2002). Human DNA polymerase lambda functionally and physically interacts with proliferating cell nuclear antigen in normal and translesion DNA synthesis. J Biol Chem 277, 48434-48440.

43. Haracska, L., Johnson, R. E., Unk, I., Phillips, B., Hurwitz, J., Prakash, L. \& Prakash, S. (2001). Physical and functional interactions of human DNA polymerase eta with PCNA. Mol Cell Biol 21, 7199-7206.

44. Haracska, L., Johnson, R. E., Unk, I., Phillips, B. B., Hurwitz, J., Prakash, L. \& Prakash, S. (2001). Targeting of human DNA polymerase iota to the replication machinery via interaction with PCNA. Proc Natl Acad Sci U S A 98, 1425614261.

45. Haracska, L., Acharya, N., Unk, I., Johnson, R. E., Hurwitz, J., Prakash, L. \& Prakash, S. (2005). A single domain in human DNA polymerase iota mediates 
interaction with PCNA: implications for translesion DNA synthesis. Mol Cell Biol 25, 1183-1190.

46. Dionne, I., Brown, N. J., Woodgate, R. \& Bell, S. D. (2008). On the mechanism of loading the PCNA sliding clamp by RFC. Mol Microbiol 68, 216-222.

47. Gulbis, J. M., Kelman, Z., Hurwitz, J., O'Donnell, M. \& Kuriyan, J. (1996). Structure of the C-terminal region of p21(WAF1/CIP1) complexed with human PCNA. Cell 87, 297-306.

48. Robbins, J. B., Murphy, M. C., White, B. A., Mackie, R. I., Ha, T. \& Cann, I. K. (2004). Functional analysis of multiple single-stranded DNA-binding proteins from Methanosarcina acetivorans and their effects on DNA synthesis by DNA polymerase BI. J Biol Chem 279, 6315-6326.

49. Hosfield, D. J., Mol, C. D., Shen, B. \& Tainer, J. A. (1998). Structure of the DNA repair and replication endonuclease and exonuclease FEN-1: coupling DNA and PCNA binding to FEN-1 activity. Cell 95, 135-146.

50. Pritchett, M.A. \& Metcalf, W.W. (2005). Genetic, physiological and biochemical characterization of multiple methanol methyltransferase isozymes in Methanosarcina acetivorans C2A. Mol Micro 56, 1183-1194.

51. Guss, A.M., Rother, M., Zhang, J.K., Kulkarni, G., \& Metcalf, W.W. (2008). New method for tightly regulated gene expression and highly efficient chromosomal integration of cloned genes for Methanosarcina species. Archaea 2, 193-203. 


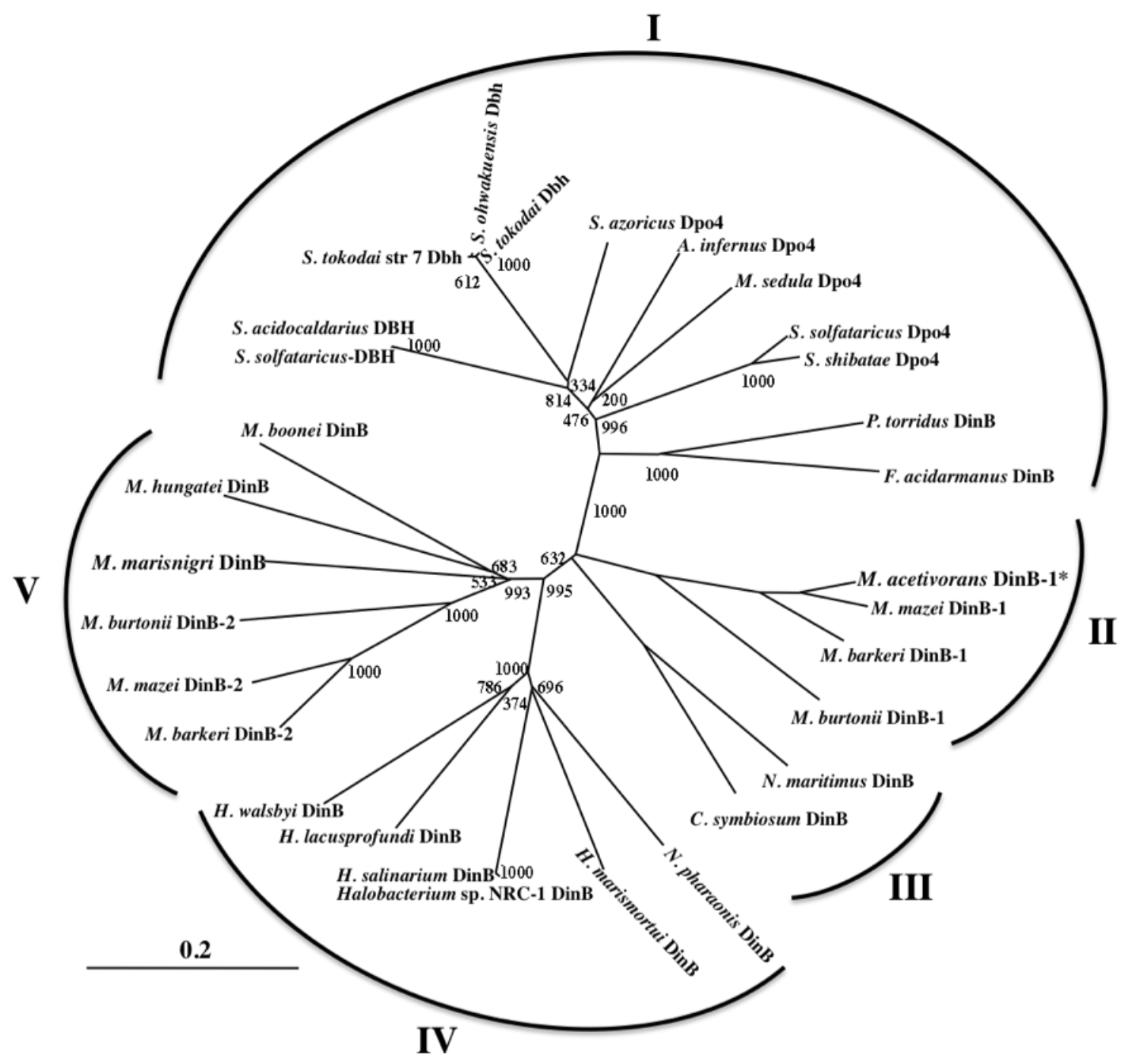

Fig. 1 
Motif I (6-33)

MacDinB-1

MmaDinB-1

MbaDinB-1

MbuDinB-1

NmaDinB

CsymDinB

MhuDinB

MboDinB

MmarisDinB

MbaDinB-2

MmaDinB-2

MbuDinB-2

HspNRC-1DinB

HsaDinB

NphaDinB

HmaDinB

HlaDinB

HwaDinB

SsoDbh

SacDbh

SsoDpo 4

SshDpo 4

StoDbh

SohDbh

Sto7Dbh

SazDbh

AinDpo 4

MseDpo 4

PtoDinB

FacDinB

MacDinB-1
MmaDinB-1
MbaDinB-1
MbuDinB-1
NmaDinB
CsymDinB
MhuDinB
MboDinB
MmarisDinB
MbaDinB-2
MmaDinB-2
MbuDinB-2
HspNRC-1DinB
HsaDinB
NphaDinB
HmaDinB
HlaDinB
HwaDinB
Sso-Dbh
SacDbh
SsoDpo4
SshDpo4
StoDbh
SohDbh
Sto7Dbh
SazDbh
AinDpo4
MseDpo4
PtoDinB
FacDinB

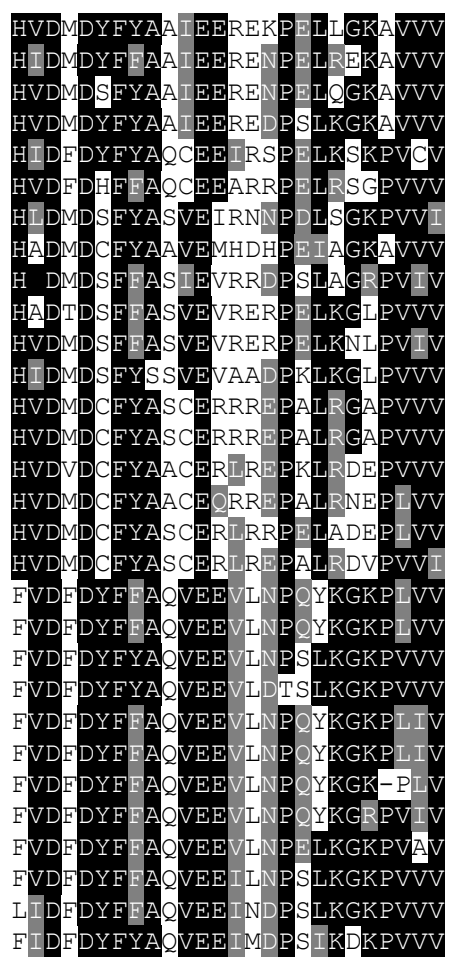

Motif II (46-61)
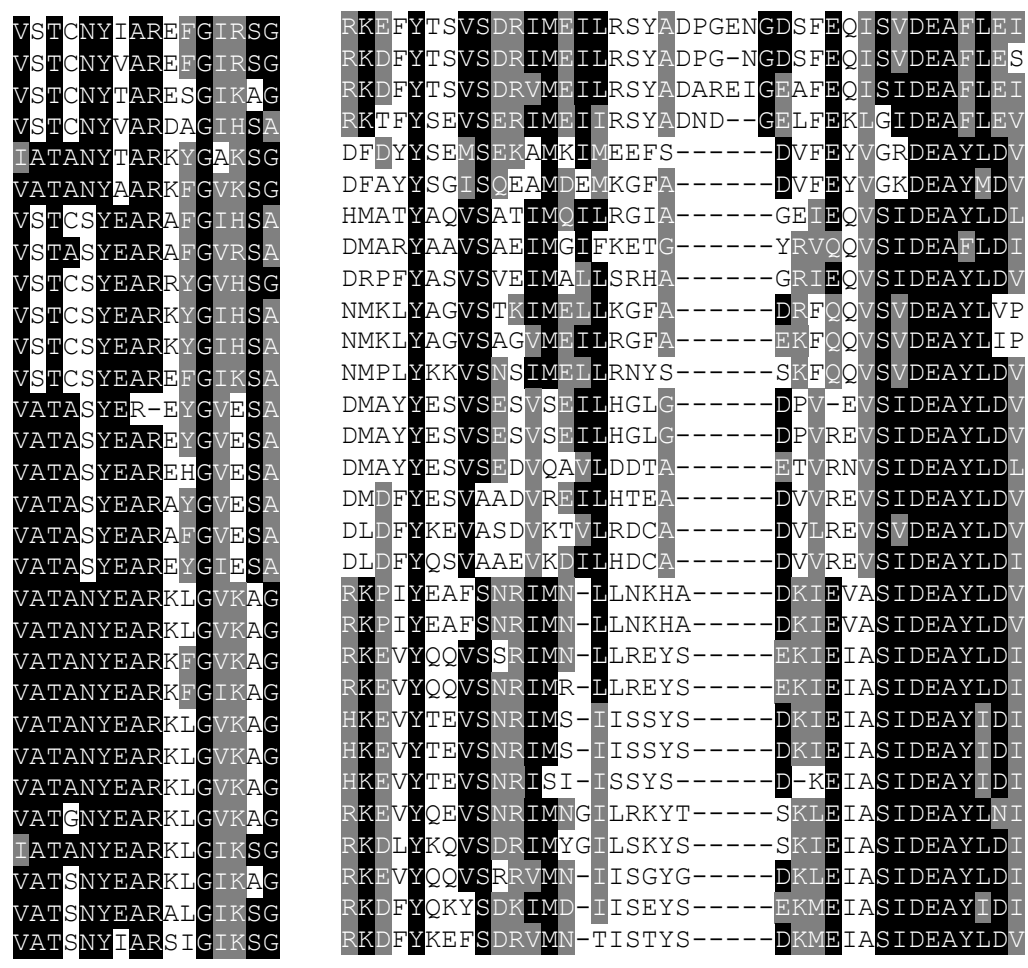

Motif IV (134-170)

Motif V (184-214)

Motif VI (360-366)

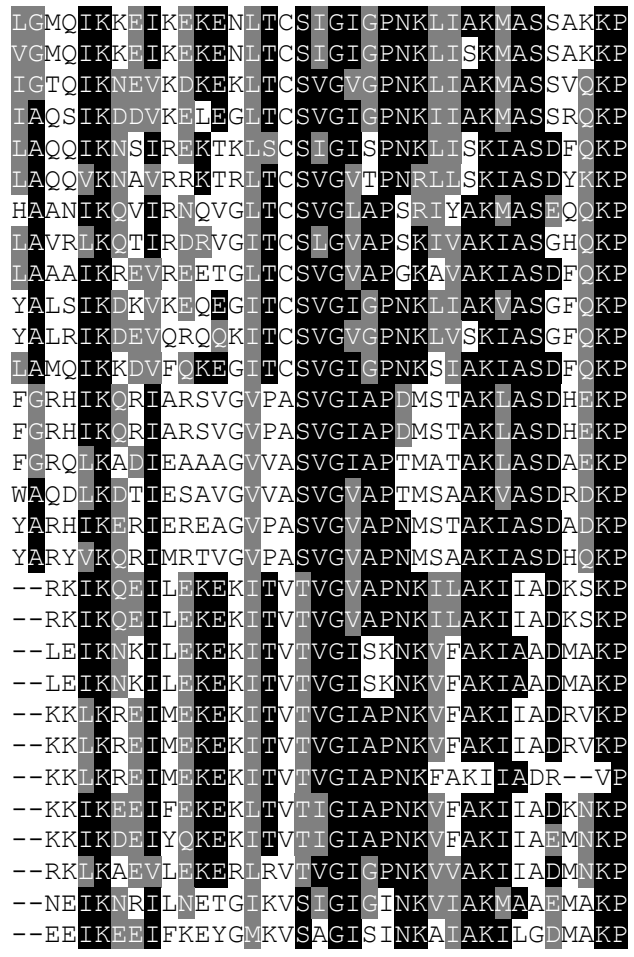

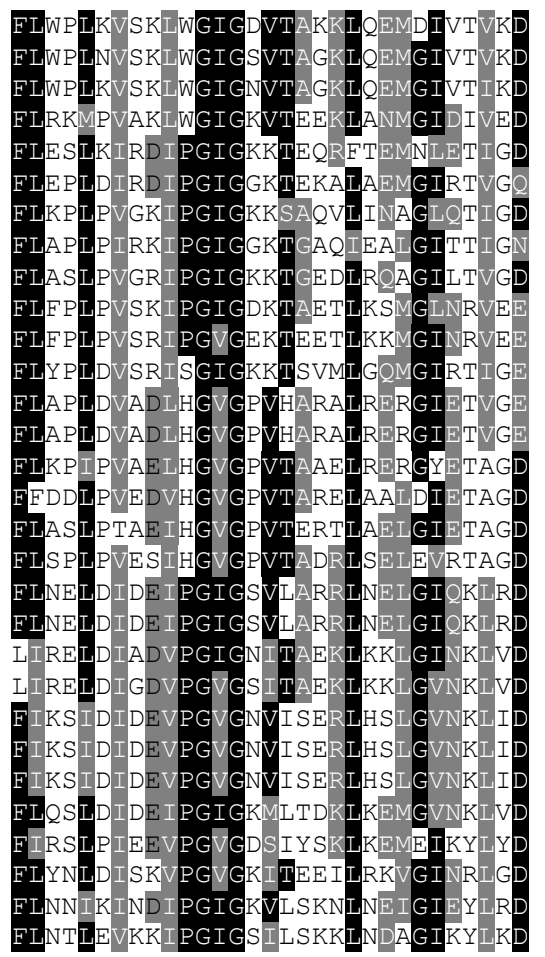

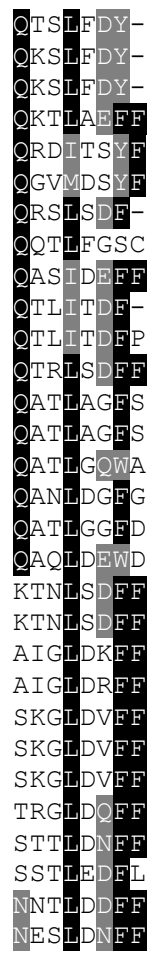

Fig. 2 


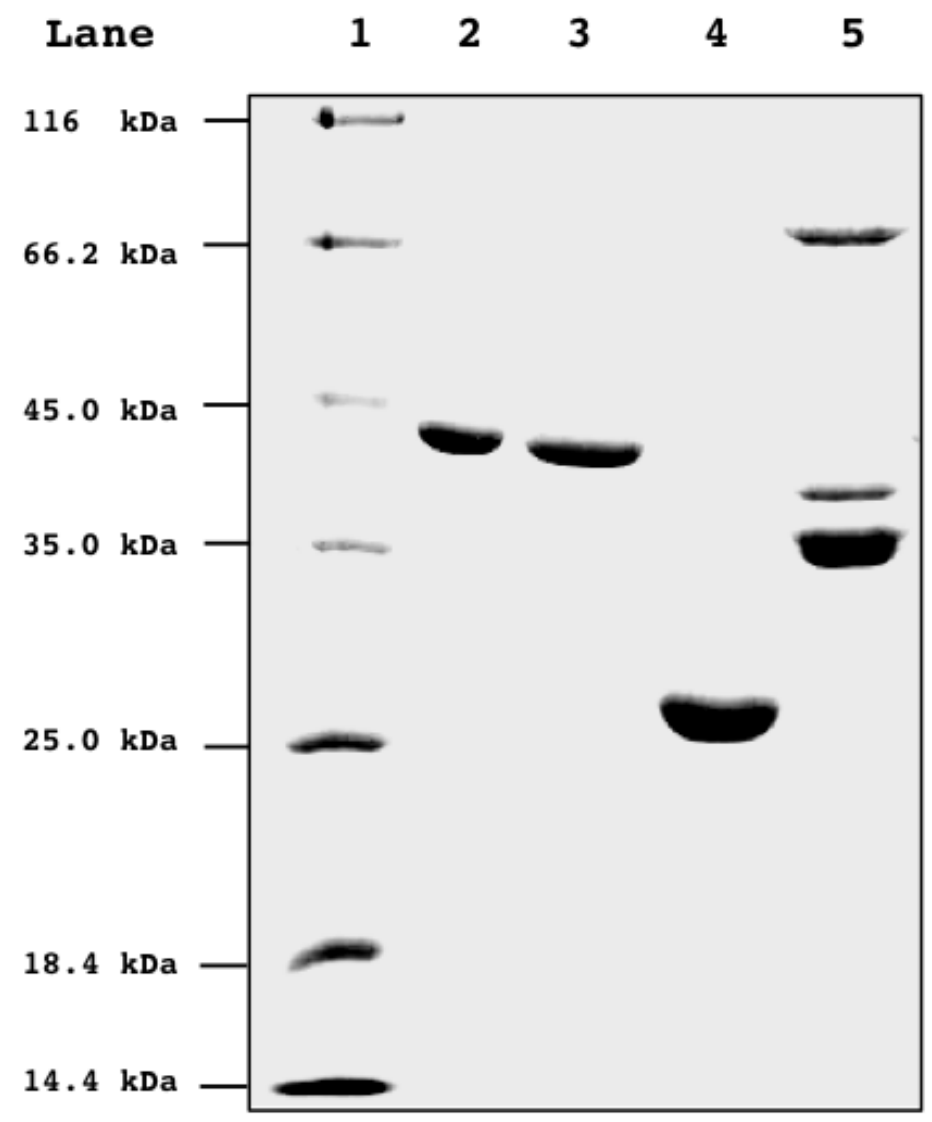

Fig. 3 
A

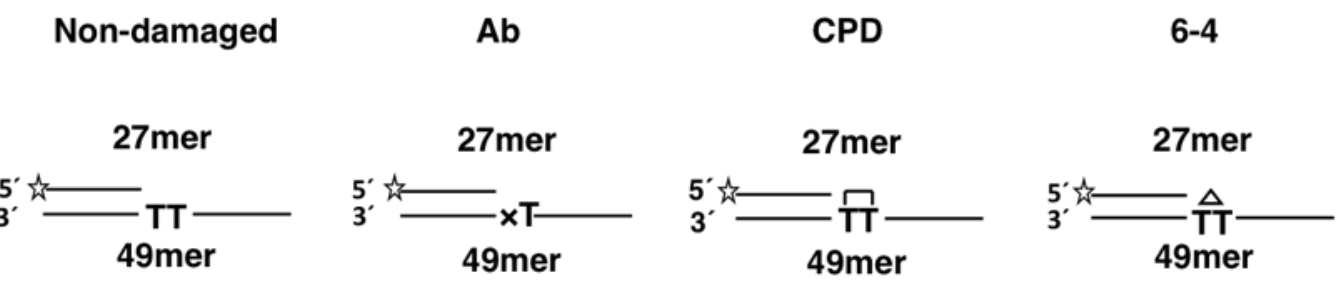

B

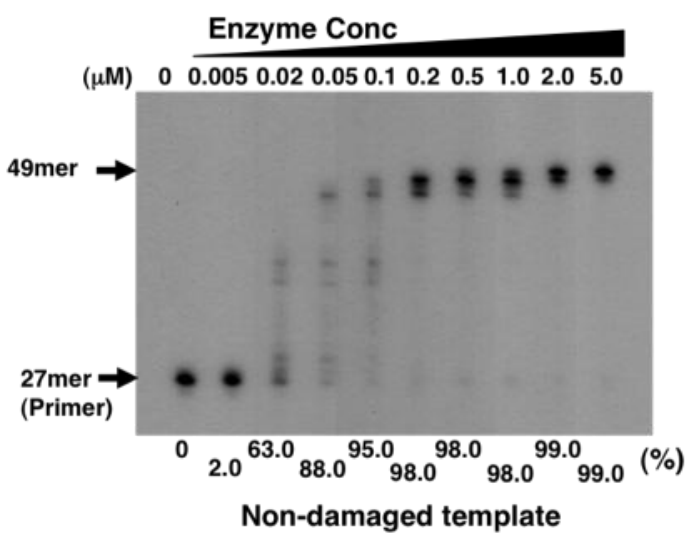

C

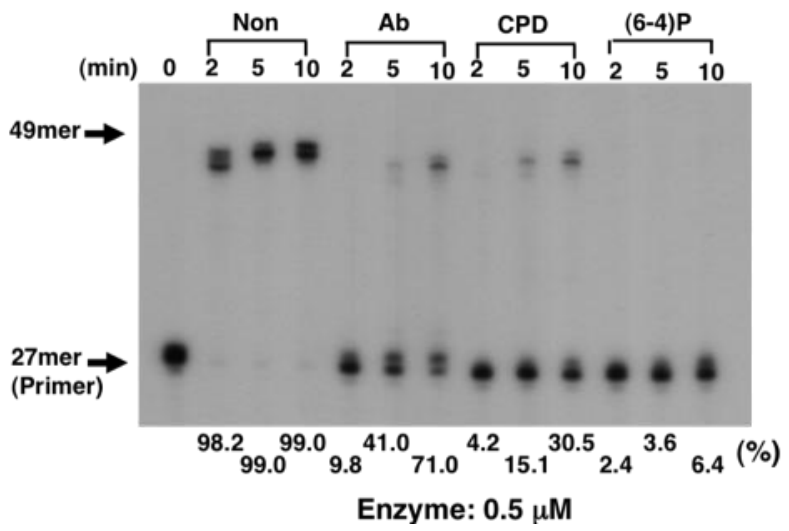

Fig. 4 


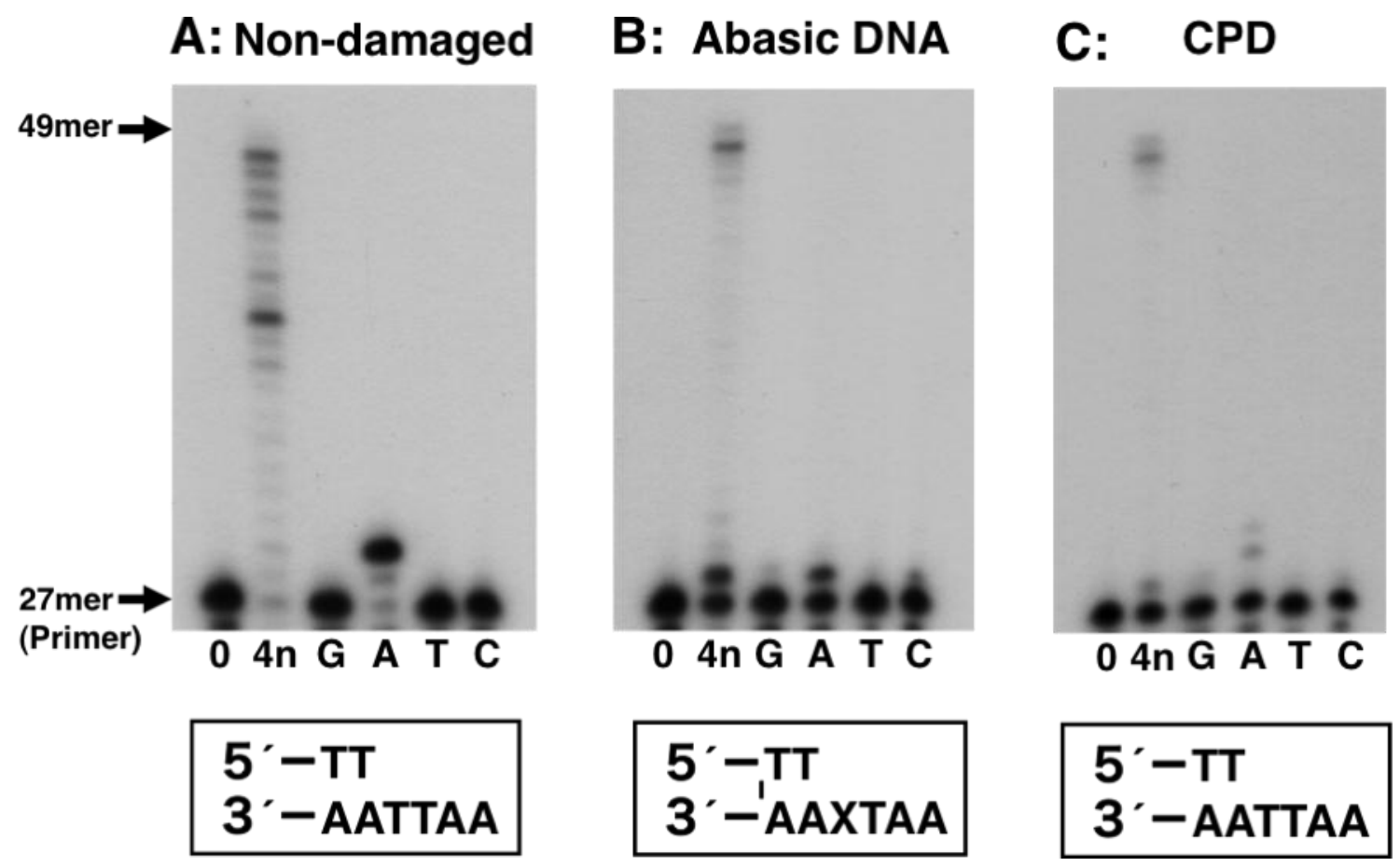

Fig. 5 
A Abasic

PCNA Conc

(nM) $P \quad 0 \quad 51020501002005001000$

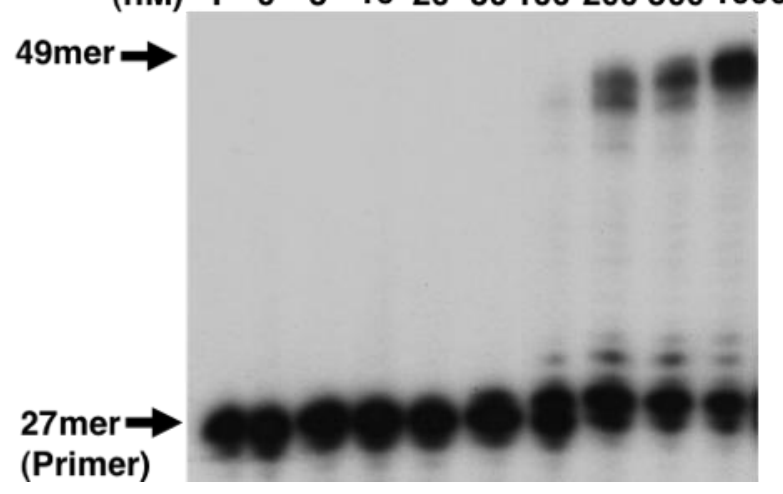

B

CPD

PCNA Conc
P 051020501002005001000

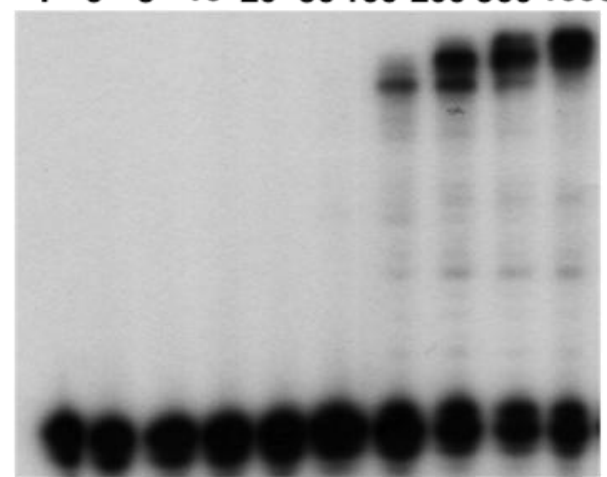

Fig. 6 

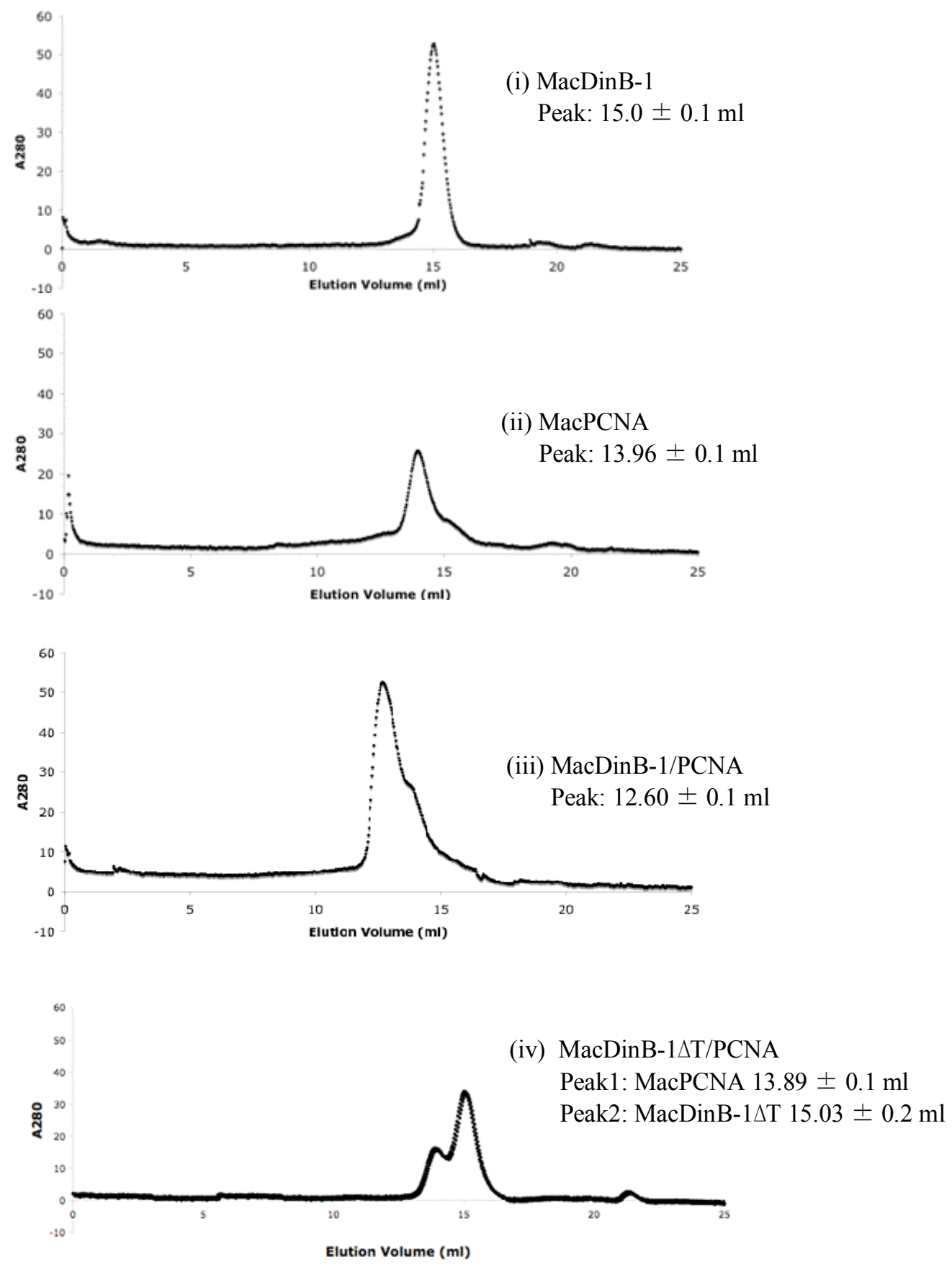

Fig. 7A 
$\begin{array}{llllllllllllll}\text { Elution Volume } \quad & 11.0 & 11.5 & 12.0 & 12.5 & 13.0 & 13.5 & 14.0 & 14.5 & 15.0 & 15.5 & 16.0 & 16.5 & (\mathrm{ml})\end{array}$

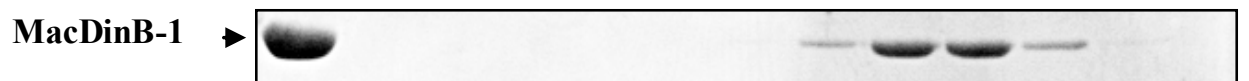

MW: 42.3 kDa

MacPCNA

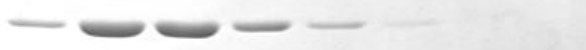

MW: 26.6 kDa
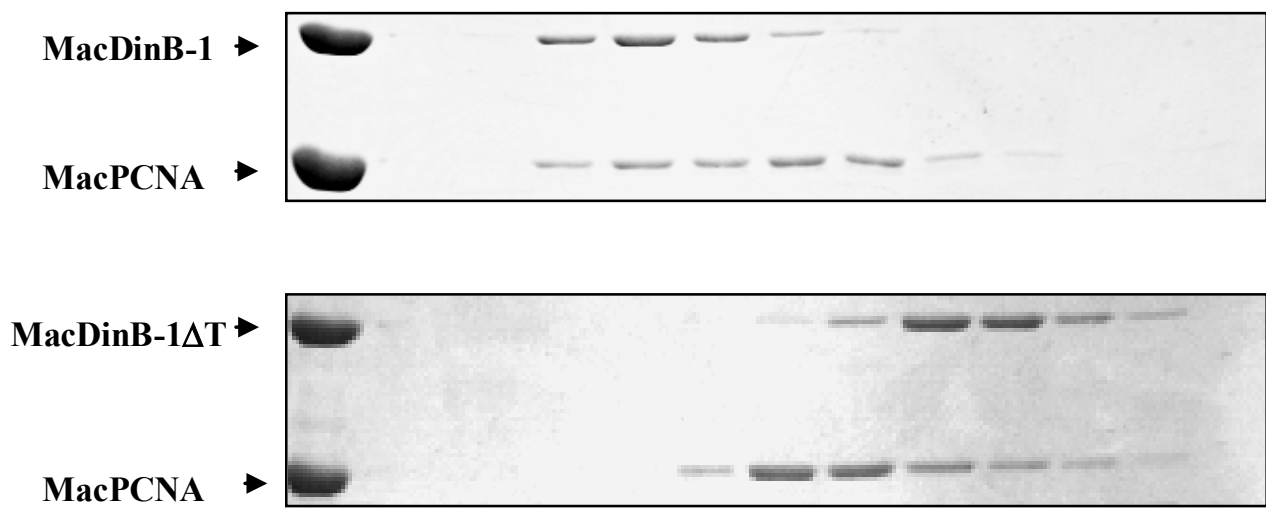

Fig. 7B 


\author{
M. acetivorans DinB \\ $M$. acetivorans PolBI-I \\ $M$. acetivorans PolBI-II \\ $M$. acetivorans RFCL-I \\ $M$. acetivorans RFCL-II \\ $M$. acetivorans FEN-I \\ $M$. acetivorans $\mathrm{Hjm}$ \\ $M$. acetivorans DP2 \\ Human FEN-I \\ Human p21 WAF1/CIP1 \\ Consensus
}

\author{
QTSLFDY \\ QKGLFDFE \\ QRSLFDF \\ QKTLNMGF \\ QKTLFDF \\ QKTLDQWF \\ QSTISDF \\ QLGLSDFM \\ QGRLDDFF \\ QTSMTDFY \\ QXXLXXF/W/Y
}

Fig. 8 
A.

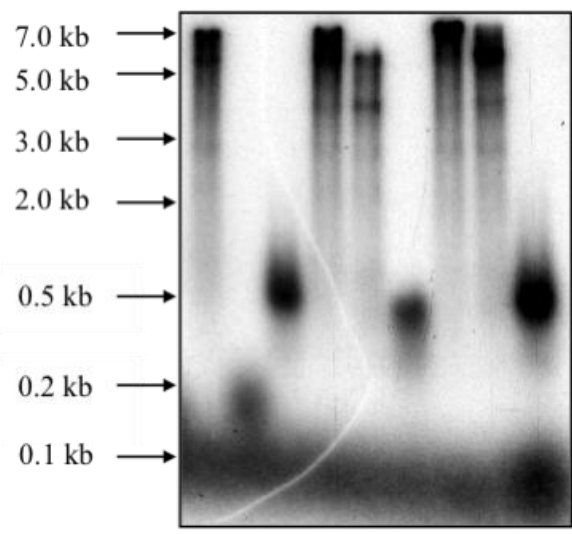

MacDinB-1 \& mutants WT $\triangle Q T$ S $T$ F MacPCNA ++++++++ $\begin{array}{llllllllll}\text { MacRFC complex } & + & + & + & + & + & + & + & + & + \\ \text { Template/Primer } & + & + & + & + & + & + & + & + & +\end{array}$

B.

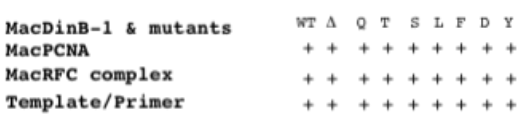

Template/Primer

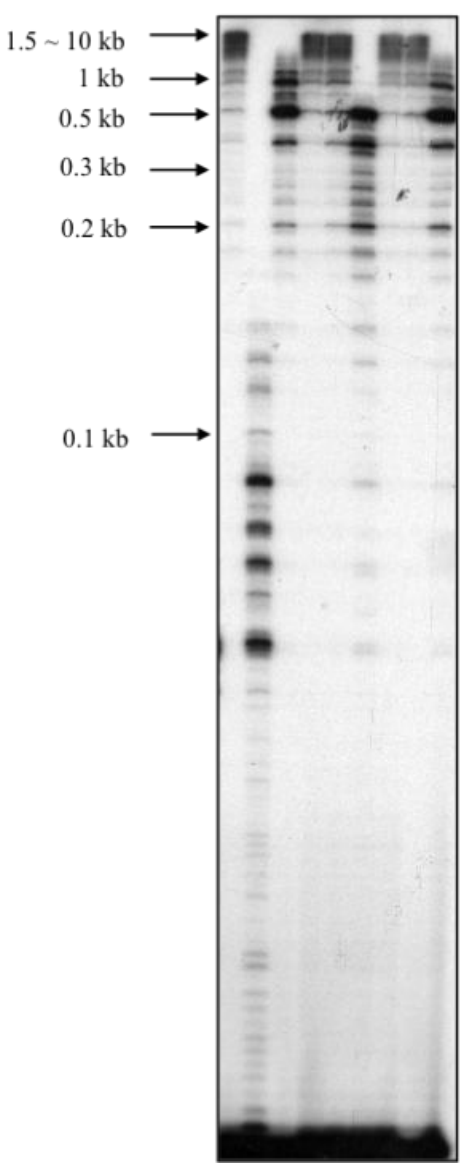

C.

MacDinB-1 MacPCNA

Template/Primer $\quad+++++$

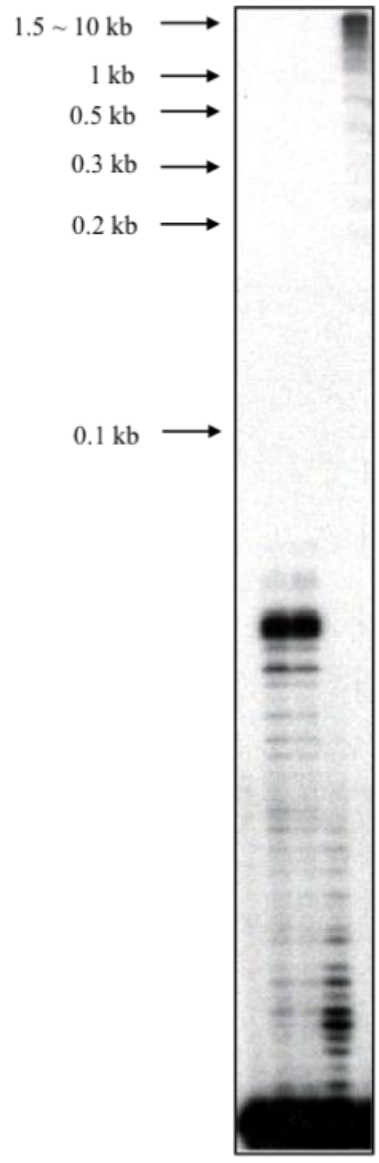

Fig. 9 
Fig. 10A

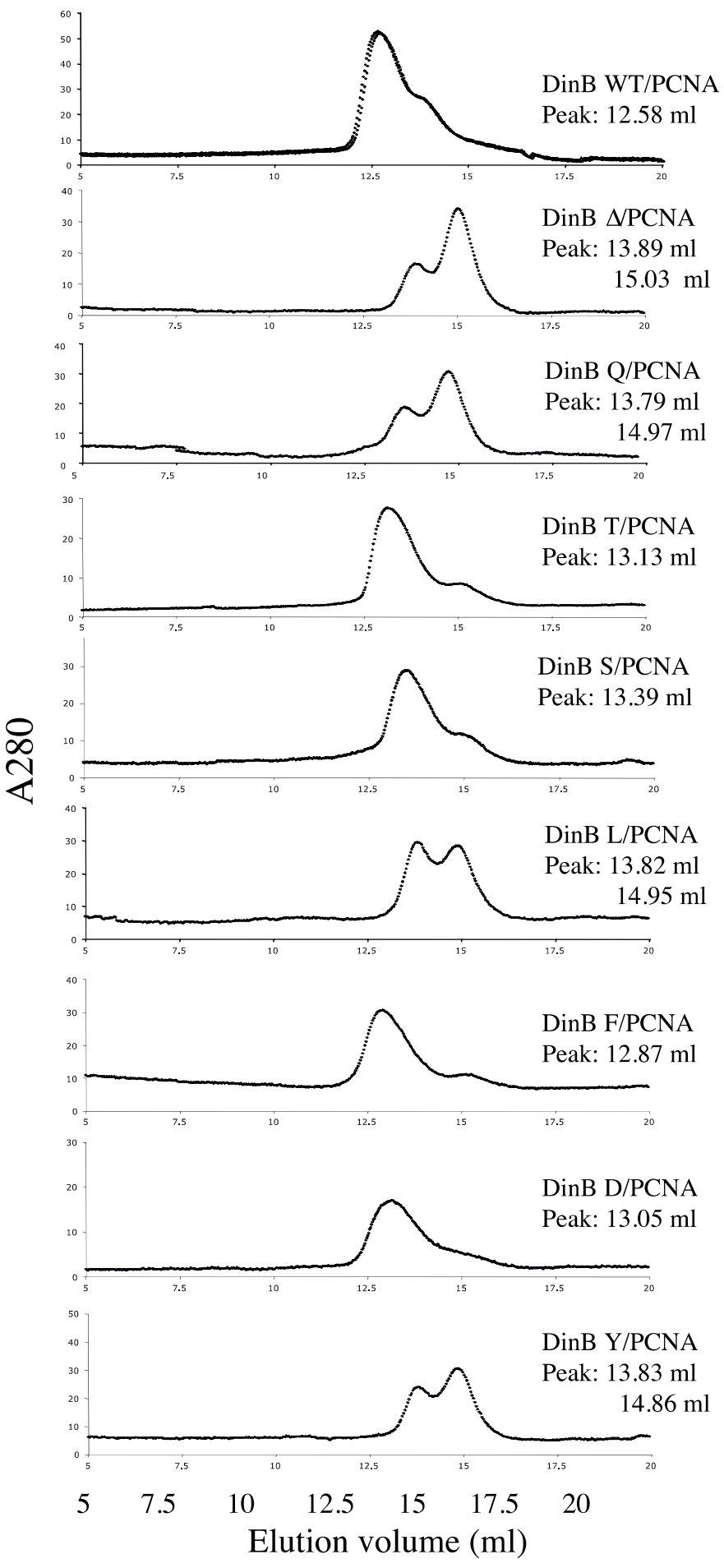




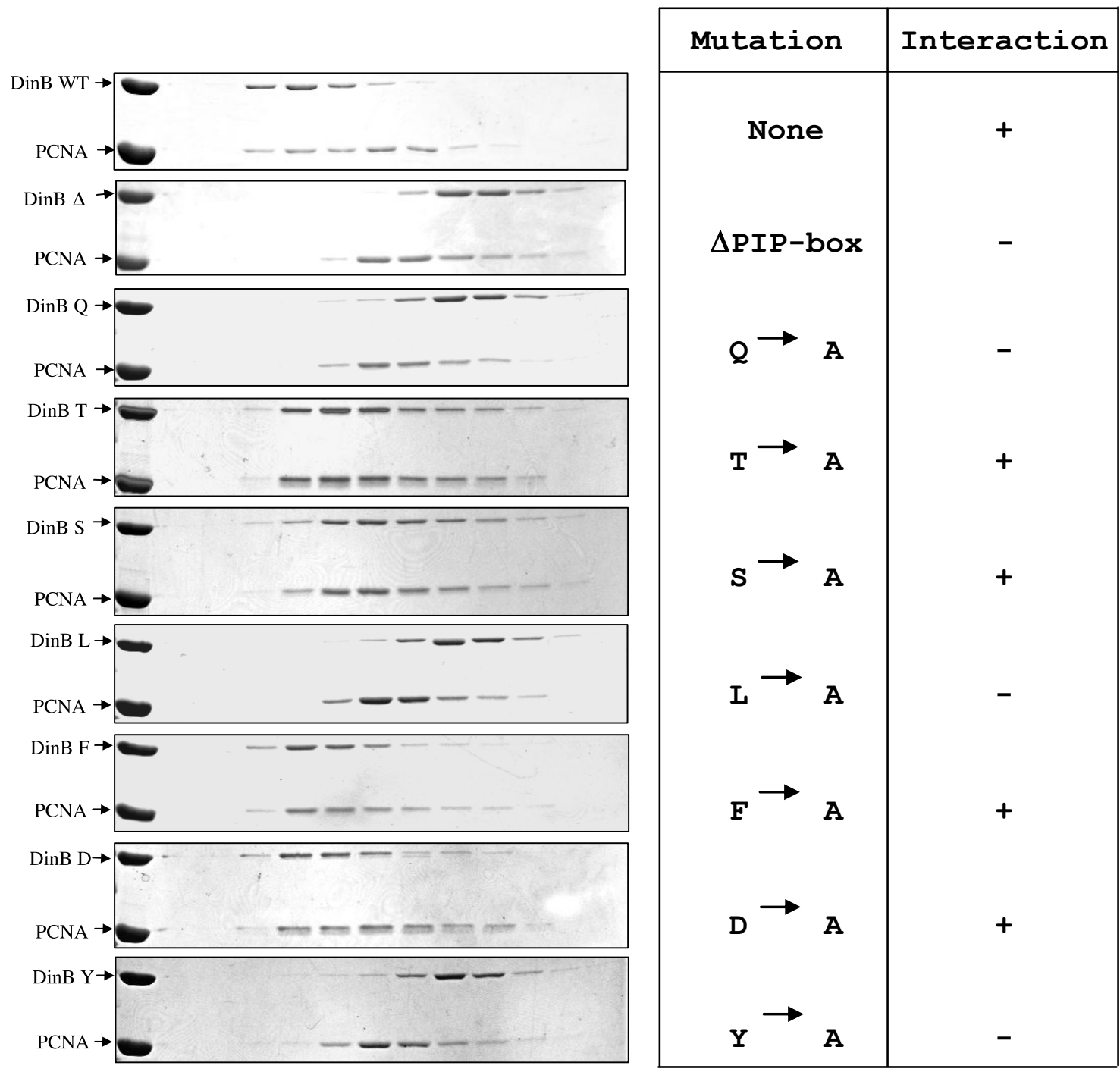

$11.011 .512 .012 .5 \quad 13.013 .514 .014 .515 .015 .516 .016 .5$ Elution Volume (ml)

Fig. 10B 


\section{Supplementary Fig. 1.}

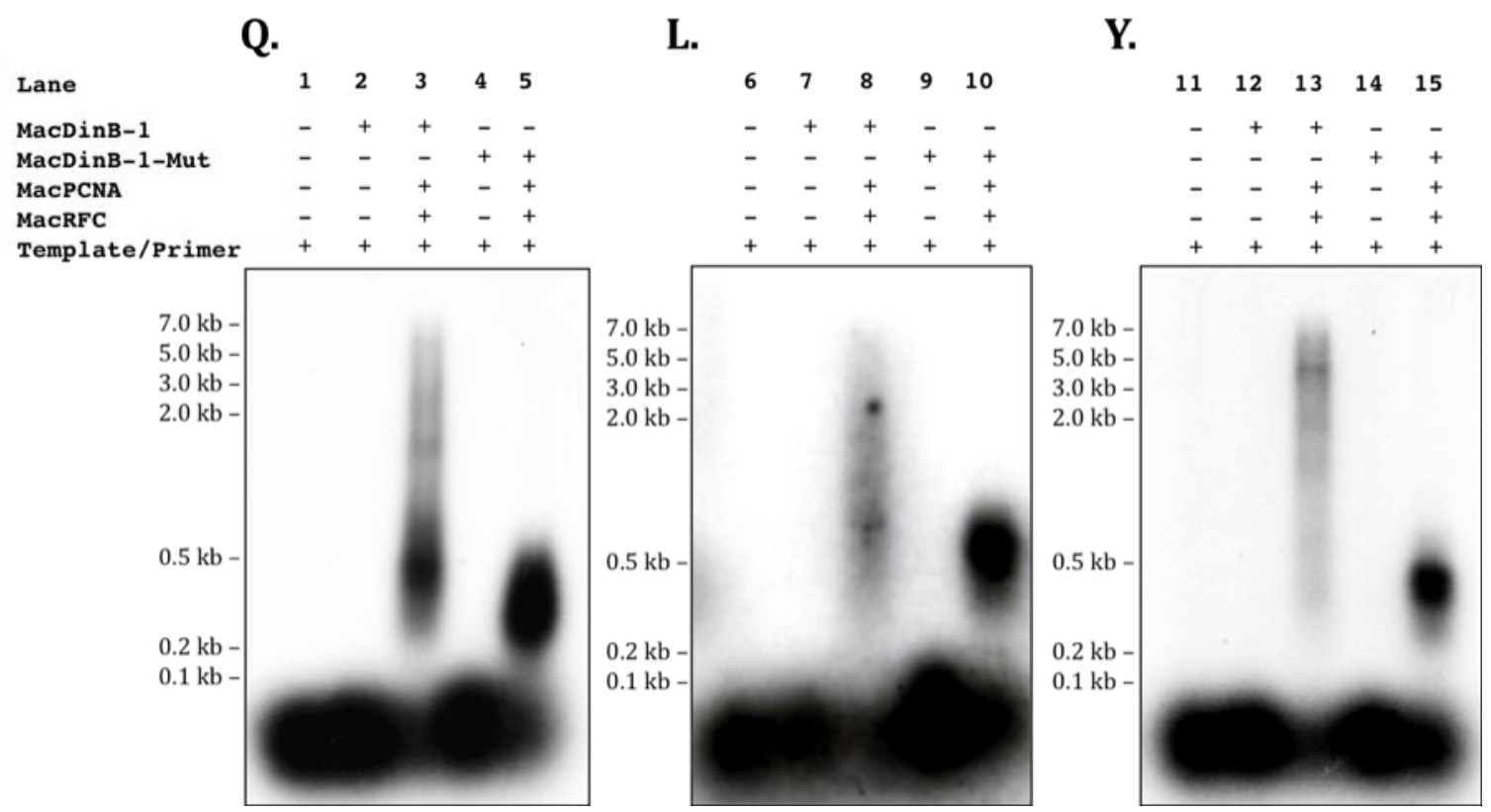

Three different mutations (Q360A, L363A, and Y366A) in the PIP-box or motif VI of MacDinB-1 impair stimulation of primer extension in the presence of accessory factors (MacPCNA and MacRFC). Lanes 1, 6, 11 are template substrate alone or negative control; lanes 2, 7, and 12 are substrate/MacDinB-1; lanes 3, 8, and 13 are subtrate/MacDinB-1/MacPCNA/MacRFC or positive control; Lanes 4, 9, and 14 are substrate/MacDinB-1 mutant; and lanes 5, 10, and 15 are substrate/MacDinB-1 mutant/MacPCNA/MacRFC. The data showed that both MacDinB-1 and its mutants synthesized very short DNA that are almost not detectable by $1 \%$ alkaline agarose gel electrophoresis (lanes 2, 7, 12 and lanes 4, 9, 14), and while MacDinB-1 synthesized long products in the presence of accessory proteins (lanes 3, 8, 13), the mutants exhibited impaired capacity to synthesize very long DNA in the presence of the accessory proteins (Lane $5,10,15$ ). The substrate for DNA synthesis was $1 \mu \mathrm{g}$ of singly-primed M13mp18 ssDNA in DNA polymerase reaction buffer as described in the Materials and Methods. The reaction mixtures were incubated at $37{ }^{\circ} \mathrm{C}$ for $30 \mathrm{~min}$, and the products were denatured by boiling and then analyzed by $1 \%$ alkali agarose gel electrophoresis. The results were visualized by autoradiography. 


\section{Supplementary Fig. 2}

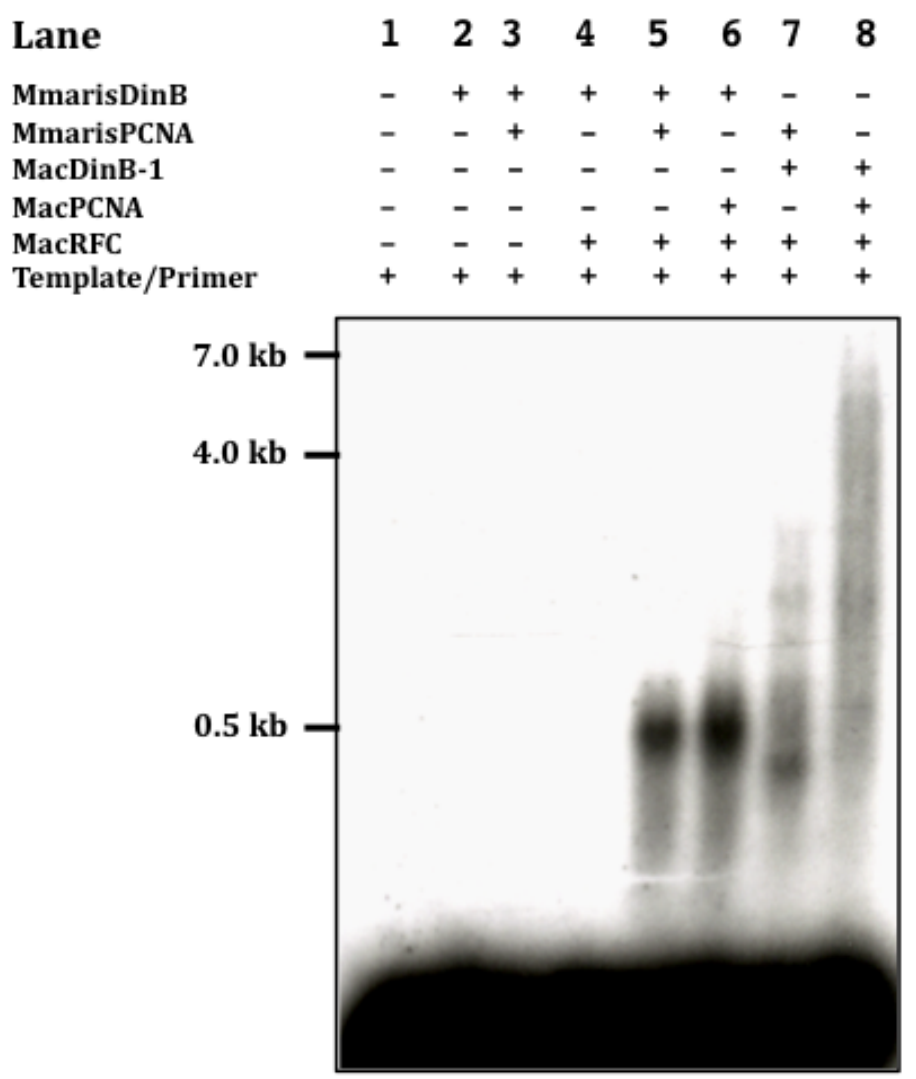

Primer extension analysis of the DinB homolog of Methanoculleus marisnigri (MmarisDinB), a member of cluster $\mathrm{V}$ in the presence of MacPCNA and MmarisDinB. Lane 1, substrate alone or negative control; lane 2, substrate/MmarisDinB; lane 3, substrate/MmarisDinB/MmarisPCNA; lane 4, substrate/MmarisDinB/MacRFC; lane $\quad 5, \quad$ substrate/MmarisDinB/MacRFC/MmarisPCNA; lane 6 , substrate/MmarisDinB/MacRFC/MacPCNA; lane $7, \quad$ substrate/MacDinB1/MmarisPCNA/MacRFC; lane 8, substrate/MacDinB-1/MacPCNA/MacRFC or positive control. The substrate for DNA synthesis was $1 \mu \mathrm{g}$ of singly-primed M13mp18 ssDNA in DNA polymerase reaction buffer. The reaction mixtures were incubated at $37{ }^{\circ} \mathrm{C}$ for 30 min, and the products were denatured by boiling and then analyzed by $1 \%$ alkali agarose gel electrophoresis. The results were visualized by autoradiography. The products synthesized by MmarisDinB, a member of cluster V, in the presence of the accessory factors (cognate or heterologous) were always shorter than the products synthesized by MacDinB-1 in the presence of the same accessory factors. 